\title{
UK Renal Registry 19th Annual Report: Chapter 3 Demographic and Biochemistry Profile of Kidney Transplant Recipients in the UK in 2015: National and Centre-specific Analyses
}

\author{
Edward Sharples ${ }^{a}$, Anna Casula ${ }^{b}$, Catherine Byrne ${ }^{c}$

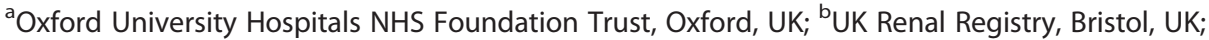 \\ ${ }^{c}$ Nottingham University Hospitals NHS Trust, Nottingham, UK
}

\section{Keywords}

Blood pressure - Bone metabolism . Chronic kidney disease . Clinical Commissioning Group • Deceased donor · eGFR . Epidemiology · Ethnicity · Graft function - Haemoglobin . Live donor - Outcomes - Renal transplantation - Survival

\section{Summary}

- There was a $1 \%$ fall in overall renal transplant numbers in 2015, with a fall in kidney donation from donors after brainstem death $(6 \%)$ and from living donors (5\%).

- In 2015, death-censored renal transplant failure rates in prevalent patients were similar to previous years at $2.7 \%$ per annum. Transplant patient death rates were similar at 2.5 per 100 patient years.

- The median age of incident and prevalent renal transplant patients in the UK was 50.9 and 53.8 years respectively.
- The median eGFR of prevalent renal transplant recipients was $51.8 \mathrm{ml} / \mathrm{min} / 1.73 \mathrm{~m}^{2}$.

- The median eGFR of patients one year after transplantation was $57.5 \mathrm{ml} / \mathrm{min} / 1.73 \mathrm{~m}^{2}$ post live transplant, $53.7 \mathrm{ml} / \mathrm{min} / 1.73 \mathrm{~m}^{2}$ post brainstem death transplant and $50.4 \mathrm{ml} / \mathrm{min} / 1.73 \mathrm{~m}^{2}$ post circulatory death transplant.

- In $2015,13.3 \%$ of prevalent transplant patients had eGFR $<30 \mathrm{ml} / \mathrm{min} / 1.73 \mathrm{~m}^{2}$.

- The median decline in eGFR slope beyond the first year after transplantation was $-0.56 \mathrm{ml} / \mathrm{min} /$ $1.73 \mathrm{~m}^{2} /$ year.

- In 2015, infection (24\%) and malignancy (22\%) remained the commonest causes of death in patients with a functioning renal transplant.

\section{KARGER}

Fax +41613061234 E-Mail karger@karger.com www.karger.com/nef
This article is licensed under the Creative Commons AttributionNonCommercial-NoDerivatives 4.0 International License (CC BYNC-ND) (http://www.karger.com/Services/OpenAccessLicense), Usage and distribution for commercial purposes as well as any distribution of modified material requires written permission
E J Sharples

UK Renal Registry, Southmead Hospital, Southmead Road,

Bristol, BS10 5NB, UK

Email: renalregistry@renalregistry.nhs.uk 


\section{Introduction}

This chapter includes independent analyses regarding renal transplant activity and survival data from the UK Transplant Registry, held by the Organ Donation and Transplantation Directorate (ODT) of NHS Blood and Transplant (NHSBT). The UK Renal Registry (UKRR) has performed additional analyses of renal transplant recipient follow-up data examining demographics, clinical and biochemical variables. NHSBT records all information regarding the episode of transplantation (donor and recipient details) and the UKRR holds additional information on key clinical and biochemical variables in renal transplant recipients. The co-operation between these two organisations results in a comprehensive database describing the clinical care delivered to renal transplant patients within the UK. This allows for the comparison of key quality measures between centres and provides insight into the processes involved in the care of such patients in the UK.

This chapter is divided into six sections: (1) transplant activity, waiting list and survival data; (2) transplant demographics; (3) clinical and laboratory outcomes; (4) analysis of prevalent patients by chronic kidney disease (CKD) stage; (5) eGFR slope analysis; and (6) cause of death in transplant recipients. Methodology, results and a discussion of these analyses are provided in detail for all six sections separately.

The UK Renal Registry methodology has previously been described [1]. The UKRR collects quarterly clinical data via an electronic data extraction process from hospital based renal IT systems on all patients receiving renal replacement therapy. Throughout the chapter, the number preceding the centre name in each figure indicates the percentage of missing data for that centre for that variable.

Unless otherwise specified, prevalent transplant patients were defined as patients with a functioning renal transplant on the 31st December 2015.

A list of the Renal Association recommended audit measures which are relevant to the transplant population are given in appendix 1 of this chapter. Several of the audit measures are not currently reported by the UKRR in the annual report; the reasons behind this are varied, but predominantly relate to a high proportion of incomplete data or that the relevant variable is not currently within the specified UKRR dataset. Over time it is hoped to work with the renal community to improve reporting across the range of recommended standards.

\section{Transplant activity, waiting list activity and survival data}

\section{Introduction}

NHSBT prospectively collects donor and recipient data at the time of transplantation. They also request that transplant centres provide an annual paper based data return on the status of the recipient including graft function. This enables ODT to generate comprehensive analyses of renal transplant activity and graft survival statistics.

NHSBT attributes a patient to the centre that performed the transplant operation irrespective of where the patient was cared for before or after the procedure and hence only reports on transplant centre performance.

\section{Methods}

In 2015, there were 23 UK adult renal transplant centres, 19 in England, two in Scotland and one each in Northern Ireland and Wales.

Annual organ-specific updates and five-year reports with comprehensive data concerning the number of patients on the transplant waiting list, percentage of pre-emptive listing, the number of transplants performed, the number of deceased kidney donors (donor after brainstem death and donor after circulatory death), living kidney donors, patient survival and graft survival are available on the NHSBT website (https://www.organdonation.nhs.uk/ statistics/)

\section{Results}

During 2015, 3,174 kidney or kidney plus transplants were performed (table 3.1). The absolute number of living kidney donors showed a small decline in 2015, but still represented $32.9 \%$ of all transplants performed. Compared to the relative fall observed in 2014, there was recovery in the number of donor after circulatory death (DCD) transplants $(+12 \%)$, whereas the number of deceased brainstem death donors did not increase. The number of kidney plus other organ transplants has not changed.

There were small differences in one- and five-year risk adjusted patient and graft survival rates amongst UK kidney transplant centres (table 3.2). These graft survival rates include grafts with primary non-function, which are excluded from analysis by some registries.

Using data from the UKRR on prevalent renal transplant patients on 1st January 2015, the death rate during 2015 was 2.5 per 100 patient years (CI 2.3-2.7) when censored for return to dialysis, and 2.7 per 100 patient years (CI 2.5-2.9) without censoring for dialysis. These death rates were similar to those observed over the last five years and have not shown any impact from the increasing age or comorbidity of the transplanted cohort. 
Table 3.1. UK kidney and kidney plus other organ transplant numbers in the UK (including paediatric), 1/1/2013-31/12/2015

\begin{tabular}{|c|c|c|c|c|}
\hline Organ & 2013 & 2014 & 2015 & \% change $2014-2015$ \\
\hline Donor after circulatory death ${ }^{\mathrm{b}}$ & 794 & 713 & 802 & 12 \\
\hline Kidney and liver ${ }^{c}$ & 11 & 12 & 21 & \\
\hline Kidney and heart & 1 & 1 & 0 & \\
\hline Kidney and pancreas ${ }^{\mathrm{d}}$ & 190 & 171 & 175 & 2 \\
\hline Total kidney transplants & 3,261 & 3,201 & 3,174 & -1 \\
\hline
\end{tabular}

${ }^{a}$ Includes en bloc kidney transplants (4 in 2013, 3 in 2014, 4 in 2015) and double kidney transplants (18 in 2013, 22 in 2014, 15 in 2015)

${ }^{b}$ Includes en bloc kidney transplants (6 in 2013, 4 in 2014, 8 in 2015) and double kidney transplants (53 in 2013, 51 in 2014, 31 in 2015)

${ }^{c}$ Includes DCD transplants (2 in 2013)

dincludes DCD transplants (36 in 2013, 47 in 2014, 50 in 2015)

Table 3.2. Risk-adjusted first adult kidney transplant only, graft and patient survival percentage rates for UK centres*

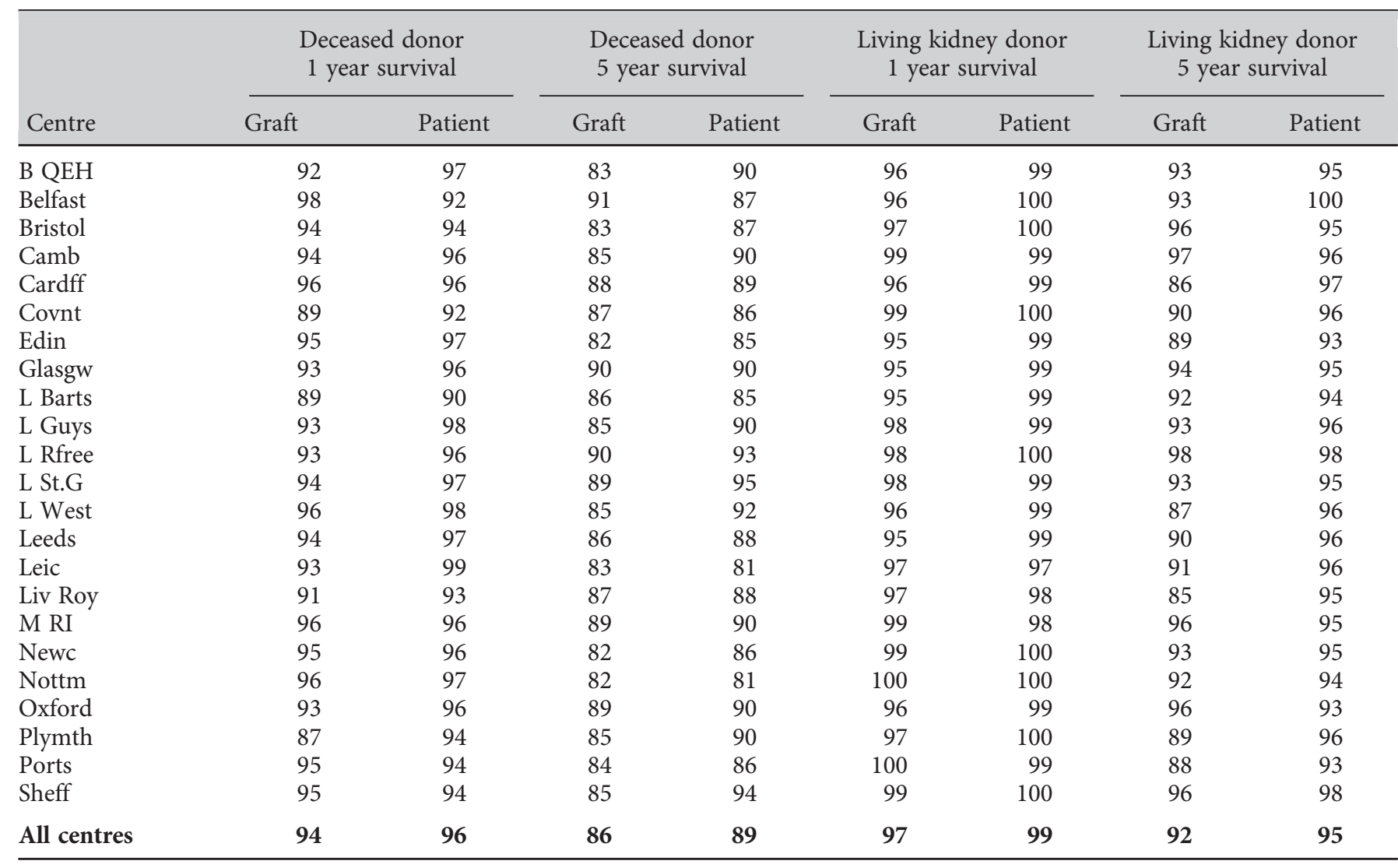

Cohorts for survival rate estimation: 1 year survival: 1/4/2010 - 31/03/2014; 5 year survival: 1/4/2006 - 31/03/2010; first grafts only re-grafts excluded for patient survival estimation. Since the cohorts to estimate 1- and 5-year survival are different, some centres may appear to have 5 year survival better than 1 year survival

*Information courtesy of NHSBT: number of transplants, patients and 95\% CI for each estimate; statistical methodology for computing risk-adjusted estimates can be obtained from the NHSBT website (see http://odt.nhs.uk/pdf/organ_specific_report_kidney_2015.pdf) 
During 2015, 2.7\% of prevalent transplant patients experienced graft failure (excluding death as a cause of graft failure), which was a slight increase on the rate in 2014 (2.4\%), and above the mean rate for 2009-2014 (2.5\%).

\section{Discussion}

During 2015, there was a $1 \%$ reduction in overall kidney transplant numbers, with a fall in the number of living kidney donors. The number of deceased donor transplants remained stable, whilst there was an increase in deceased cardiac death kidney transplants compared to 2014. The graft failure rate of $2.7 \%$ per annum and the patient death rate of 2.5 per 100 patient years are similar to previous years, despite the changes in donor and recipient populations.

\section{Transplant demographics}

\section{Introduction}

Since 2008, all UK renal centres have established electronic linkage to the UKRR or Scottish Renal Registry, giving the UKRR complete coverage of individual patient level data across the UK.

The following sections should be interpreted in the context of centre-specific variations in repatriation policies; some transplant centres continue to follow up and report on all patients they transplant, whereas others refer patients back to non-transplanting centres at some point post-transplant. Some transplant centres only refer back patients when their graft is failing. The time post-transplantation that a patient is referred back to their local centre varies between transplant centres, but the UKRR can detect duplicate patients (being reported from both transplant and referring centres) and in such situations care is usually attributed to the referring centre (see appendix B for allocation procedure). This process may result in some discrepancies in transplant numbers particularly in Oxford/Reading and Clywd/Liverpool Royal.

\section{Methods}

Cambridge renal centre (Addenbrooke's) was unable to submit the 2015 data at patient level on time for the end of 2015 UKRR data collection. The centre was able to submit summary numbers of patients still on renal replacement therapy (RRT) at the end of 2015, by treatment modality, and incident numbers. Cambridge renal centre is therefore excluded from all centre level prevalent analysis. However their data have been included in the transplant rates calculation in England and UK, where only summary numbers are needed. For the calculation of transplant rates by Clinical Commissioning Groups (CCG) or Health Board/Social Care Areas (HB), where patient-level information are needed for age/gender standardisation, Cambridge data from 2014 were used instead, which will cause a slight underestimation of the rates. Those CCGs that are at least in part covered by Addenbrooke's were identified using 2014 data and they are flagged in table 3.4 (in CCGs where between 10-70\% of the RRT population was seen in Addenbrooke's, rates are shown but the CCG is flagged, while for the two CCGs where most patients $(>70 \%)$ are thought to be seen in Addenbrooke's, rates have been blanked as they would represent mainly 2014 data.

As Colchester did not have any transplant patients they were excluded from some of the analyses, though their dialysis patients were included in the relevant dialysis population denominators. Also, this year Bangor directly submitted its data on transplant patients (previously submitted mainly by Liverpool Royal) and it is therefore now included separately in centre analyses.

For the analysis of primary renal diagnosis (PRD) in transplant recipients, a few centres were excluded from some of the incidence years because of concerns relating to the reliability of PRD coding (with these centres submitting a high percentage of uncertain or missing aetiology codes).

Information on patient demographics (age, gender, ethnicity and PRD) for patients in a given renal centre was obtained from UKRR patient registration data fields. Individual patients were assigned to the centre that returned data for them during 2015 . The prevalence of transplant patients in areas covered by individual CCG or HB was estimated based on the postcode of the registered address for patients on RRT. Data on ethnic origin, supplied as Patient Administration System (PAS) codes, were retrieved from fields within renal centre IT systems. For the purpose of this analysis, patients were grouped into White, South Asian, Black, Other and Unknown categories. The details of ethnicity regrouping into the above categories are provided in appendix $\mathrm{H}$ : Coding https://www.renalreg.org/publications-reports/.

\section{Results and Discussion}

Prevalent transplant numbers across the UK are described in table 3.3.

Table 3.3. The prevalence per million population (pmp) of renal transplants in adults in the UK on $31 / 12 / 2015$, by country

\begin{tabular}{lccccc}
\hline & England & N Ireland & Scotland & Wales & UK \\
\hline Number of prevalent transplant patients & 27,246 & 994 & 2,709 & 1,675 & 32,624 \\
Total population, mid-2015 estimates* (millions) & 54.8 & 1.9 & 5.4 & 3.1 & 65.1 \\
Prevalence transplant rate (pmp) & 497 & 537 & 504 & 540 & 501 \\
\hline
\end{tabular}

*Data from the Office of National Statistics, National Records of Scotland and the Northern Ireland Statistics and Research Agency - based on the 2011 census 
Table 3.4. The prevalence per million population ( $\mathrm{pmp}$ ) of patients with a renal transplant and standardised rate ratio in the UK, as on 31st December 2011-2015, by CCG/HB

CCG/HB - CCG in England, Health and Social Care Areas in Northern Ireland, Local Health Boards in Wales and Health Boards in Scotland $\mathrm{O} / \mathrm{E}$ - age and gender standardised transplant prevalence rate ratio

LCL - lower 95\% confidence limit

UCL - upper 95\% confidence limit

pmp - per million population

CCG/HBs with significantly high average rate ratios are bold in greyed areas

CCG/HBs with significantly low average rate ratios are italicised in greyed areas

Mid-2015 population data at CCG/HB level was obtained from the Office for National Statistics, National Records of Scotland and the Northern Ireland Statistics and Research Agency - based on the 2011 census

$\%$ non-White - percentage of the CCG/HB population that is non-White, from 2011 Census

${ }^{*}$ CCGs where at least $10 \%$ of the RRT population was seen in Cambridge renal centre. In these CCGs the rate is underestimated. In the CCGs with $>70 \%$ RRT population covered by Cambridge, the rates for 2015 have been blanked (see methods for details)

\begin{tabular}{|c|c|c|c|c|c|c|c|c|c|c|c|}
\hline UK area & CCG/HB & $\begin{array}{c}\text { Total } \\
\text { population }\end{array}$ & \multicolumn{4}{|c|}{$\mathrm{O} / \mathrm{E}$} & $\mathrm{O} / \mathrm{E}$ & $\begin{array}{l}95 \% \\
\text { LCL } \\
\end{array}$ & $\begin{array}{l}95 \% \\
\text { UCL } \\
\end{array}$ & $\begin{array}{c}\text { Crude } \\
\text { rate } \\
\text { pmp } \\
\end{array}$ & $\begin{array}{c}\% \\
\text { non- } \\
\text { White }\end{array}$ \\
\hline \multirow{6}{*}{$\begin{array}{l}\text { Cheshire, } \\
\text { Warrington } \\
\text { and Wirral }\end{array}$} & NHS Eastern Cheshire & 196,500 & 0.87 & 0.88 & 0.88 & 0.87 & 0.88 & 0.72 & 1.07 & 478 & 3.7 \\
\hline & NHS South Cheshire & 178,900 & 0.89 & 0.87 & 0.91 & 0.97 & 0.99 & 0.80 & 1.21 & 520 & 2.9 \\
\hline & NHS Vale Royal & 102,900 & 0.69 & 0.72 & 0.75 & 0.73 & 0.77 & 0.57 & 1.04 & 408 & 2.1 \\
\hline & NHS Warrington & 207,700 & 0.87 & 0.89 & 0.97 & 0.94 & 0.89 & 0.73 & 1.09 & 462 & 4.1 \\
\hline & NHS West Cheshire & 231,000 & 0.95 & 0.94 & 0.94 & 0.95 & 0.87 & 0.72 & 1.05 & 455 & 2.8 \\
\hline & NHS Wirral & 320,900 & 0.86 & 0.81 & 0.78 & 0.75 & 0.74 & 0.62 & 0.88 & 383 & 3.0 \\
\hline \multirow{5}{*}{$\begin{array}{l}\text { Durham, } \\
\text { Darlington } \\
\text { and Tees }\end{array}$} & NHS Darlington & 105,400 & 0.95 & 0.92 & 0.96 & 0.99 & 0.96 & 0.73 & 1.26 & 493 & 3.8 \\
\hline & NHS Durham Dales, Easington and Sedgefield & 274,000 & 1.05 & 1.02 & 1.04 & 1.09 & 1.04 & 0.89 & 1.22 & 555 & 1.2 \\
\hline & NHS Hartlepool and Stockton-on-Tees & 287,300 & 1.03 & 1.03 & 1.01 & 1.02 & 1.01 & 0.86 & 1.18 & 508 & 4.4 \\
\hline & NHS North Durham & 245,700 & 0.95 & 0.93 & 0.89 & 0.85 & 0.82 & 0.68 & 1.00 & 423 & 2.5 \\
\hline & NHS South Tees & 274,800 & 1.38 & 1.35 & 1.27 & 1.25 & 1.22 & 1.05 & 1.42 & 608 & 6.7 \\
\hline \multirow{12}{*}{$\begin{array}{l}\text { Greater } \\
\text { Manchester }\end{array}$} & NHS Bolton & 281,600 & 1.26 & 1.28 & 1.23 & 1.21 & 1.27 & 1.09 & 1.47 & 621 & 18.1 \\
\hline & NHS Bury & 187,900 & 1.00 & 1.01 & 0.96 & 1.00 & 1.05 & 0.86 & 1.27 & 527 & 10.8 \\
\hline & NHS Central Manchester & 188,900 & 1.06 & 1.07 & 1.12 & 1.18 & 1.29 & 1.05 & 1.58 & 487 & 48.0 \\
\hline & NHS Heywood, Middleton \& Rochdale & 214,200 & 1.09 & 1.10 & 1.09 & 0.95 & 1.01 & 0.83 & 1.22 & 490 & 18.3 \\
\hline & NHS North Manchester & 178,700 & 0.85 & 0.91 & 0.95 & 0.95 & 0.99 & 0.79 & 1.25 & 414 & 30.8 \\
\hline & NHS Oldham & 230,800 & 1.04 & 1.01 & 1.07 & 1.02 & 1.07 & 0.89 & 1.28 & 507 & 22.5 \\
\hline & NHS Salford & 245,600 & 0.95 & 1.03 & 0.97 & 1.00 & 1.02 & 0.85 & 1.22 & 476 & 9.9 \\
\hline & NHS South Manchester & 162,700 & 0.82 & 0.87 & 0.86 & 0.90 & 0.92 & 0.72 & 1.17 & 399 & 19.6 \\
\hline & NHS Stockport & 288,700 & 0.97 & 0.95 & 0.94 & 0.92 & 0.94 & 0.80 & 1.11 & 485 & 7.9 \\
\hline & NHS Tameside and Glossop & 254,900 & 1.10 & 1.06 & 1.03 & 1.06 & 1.04 & 0.88 & 1.23 & 534 & 8.2 \\
\hline & NHS Trafford & 233,300 & 0.85 & 0.88 & 0.90 & 0.94 & 0.96 & 0.80 & 1.16 & 480 & 14.5 \\
\hline & NHS Wigan Borough & 322,000 & 1.05 & 1.07 & 1.10 & 1.06 & 1.02 & 0.88 & 1.19 & 534 & 2.7 \\
\hline \multirow[t]{8}{*}{ Lancashire } & NHS Blackburn with Darwen & 146,800 & 0.97 & 0.99 & 1.01 & 1.06 & 1.07 & 0.86 & 1.35 & 504 & 30.8 \\
\hline & NHS Blackpool & 139,600 & 0.83 & 0.93 & 1.04 & 1.05 & 1.03 & 0.82 & 1.29 & 537 & 3.3 \\
\hline & NHS Chorley and South Ribble & 172,500 & 0.95 & 0.90 & 0.94 & 0.95 & 0.97 & 0.79 & 1.20 & 510 & 2.9 \\
\hline & NHS East Lancashire & 374,200 & 1.08 & 1.04 & 1.05 & 1.03 & 1.04 & 0.91 & 1.20 & 532 & 11.9 \\
\hline & NHS Fylde \& Wyre & 167,900 & 0.82 & 0.85 & 0.84 & 0.80 & 0.87 & 0.70 & 1.08 & 476 & 2.1 \\
\hline & NHS Greater Preston & 202,800 & 0.81 & 0.88 & 0.85 & 0.86 & 0.87 & 0.70 & 1.07 & 424 & 14.7 \\
\hline & NHS Lancashire North & 161,500 & 0.84 & 0.80 & 0.77 & 0.77 & 0.77 & 0.60 & 0.99 & 384 & 4.0 \\
\hline & NHS West Lancashire & 112,700 & 0.86 & 0.88 & 0.84 & 0.81 & 0.84 & 0.63 & 1.11 & 435 & 1.9 \\
\hline \multirow[t]{6}{*}{ Merseyside } & NHS Halton & 126,500 & 1.00 & 1.04 & 0.99 & 1.02 & 1.01 & 0.79 & 1.28 & 514 & 2.2 \\
\hline & NHS Knowsley & 147,200 & 0.97 & 0.97 & 0.97 & 0.91 & 0.89 & 0.69 & 1.13 & 441 & 2.8 \\
\hline & NHS Liverpool & 478,600 & 0.95 & 0.95 & 0.98 & 0.98 & 0.94 & 0.82 & 1.08 & 443 & 11.1 \\
\hline & NHS South Sefton & 158,600 & 0.91 & 0.95 & 0.91 & 0.89 & 0.87 & 0.69 & 1.09 & 454 & 2.2 \\
\hline & NHS Southport and Formby & 115,100 & 0.72 & 0.63 & 0.73 & 0.69 & 0.70 & 0.52 & 0.94 & 374 & 3.1 \\
\hline & NHS St Helens & 177,600 & 0.85 & 0.82 & 0.85 & 0.92 & 0.89 & 0.72 & 1.11 & 467 & 2.0 \\
\hline
\end{tabular}


Table 3.4. Continued

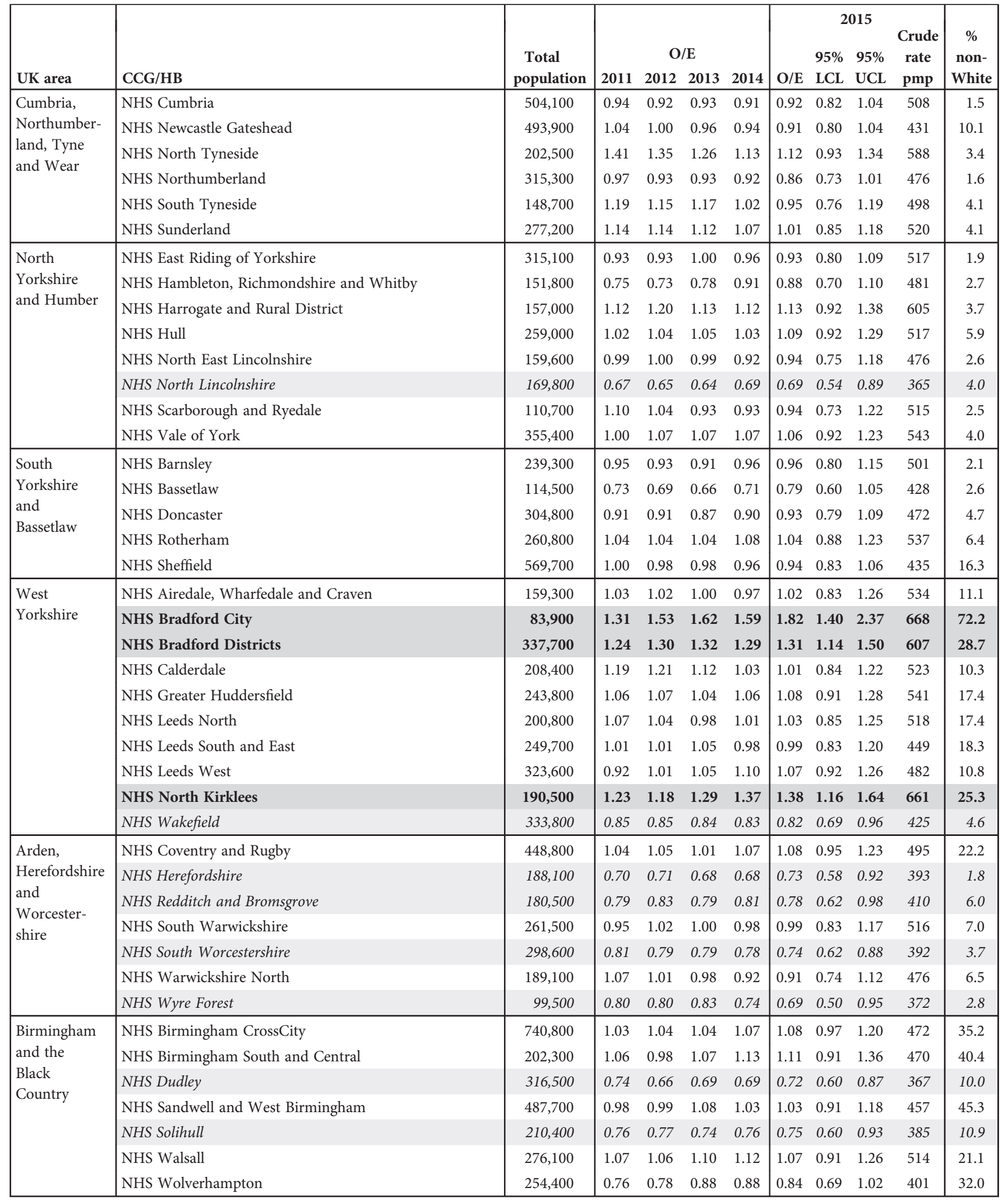


Table 3.4. Continued

\begin{tabular}{|c|c|c|c|c|c|c|c|c|c|c|c|}
\hline UK area & CCG/HB & $\begin{array}{c}\text { Total } \\
\text { population }\end{array}$ & 2011 & \multicolumn{3}{|c|}{$\mathrm{O} / \mathrm{E}$} & $O / E$ & $\begin{array}{l}95 \% \\
\text { LCL }\end{array}$ & $\begin{array}{l}95 \% \\
\text { UCL }\end{array}$ & $\begin{array}{c}\text { Crude } \\
\text { rate } \\
\text { pmp }\end{array}$ & $\begin{array}{c}\% \\
\text { non- } \\
\text { White }\end{array}$ \\
\hline \multirow{10}{*}{$\begin{array}{l}\text { Derbyshire } \\
\text { and } \\
\text { Nottingham- } \\
\text { shire }\end{array}$} & NHS Erewash & 96,300 & 0.65 & 0.64 & 0.80 & 0.85 & 0.81 & 0.59 & 1.10 & 415 & 3.2 \\
\hline & NHS Hardwick & 110,500 & 0.66 & 0.62 & 0.54 & 0.60 & 0.64 & 0.47 & 0.88 & 344 & 1.8 \\
\hline & NHS Mansfield \& Ashfield & 196,400 & 0.98 & 1.05 & 1.04 & 1.04 & 0.98 & 0.81 & 1.19 & 509 & 2.5 \\
\hline & NHS Newark \& Sherwood & 118,700 & 1.09 & 1.15 & 1.16 & 1.16 & 1.10 & 0.87 & 1.39 & 590 & 2.4 \\
\hline & NHS North Derbyshire & 272,900 & 0.78 & 0.84 & 0.78 & 0.74 & 0.75 & 0.62 & 0.90 & 410 & 2.5 \\
\hline & NHS Nottingham City & 318,900 & 0.93 & 0.96 & 0.98 & 0.96 & 0.99 & 0.84 & 1.18 & 417 & 28.5 \\
\hline & NHS Nottingham North \& East & 149,500 & 0.90 & 0.91 & 0.91 & 0.80 & 0.81 & 0.63 & 1.03 & 421 & 6.2 \\
\hline & NHS Nottingham West & 112,300 & 1.04 & 1.01 & 1.04 & 1.05 & 1.09 & 0.85 & 1.39 & 570 & 7.3 \\
\hline & NHS Rushcliffe & 114,500 & 0.88 & 0.87 & 0.94 & 0.86 & 0.78 & 0.59 & 1.04 & 411 & 6.9 \\
\hline & NHS Southern Derbyshire & 523,800 & 0.93 & 0.94 & 0.96 & 0.95 & 0.97 & 0.85 & 1.09 & 487 & 11.0 \\
\hline \multirow[t]{8}{*}{ East Anglia } & NHS Cambridgeshire and Peterborough* & 876,400 & 0.97 & 0.95 & 0.94 & 0.94 & 0.90 & 0.82 & 1.00 & 447 & 9.5 \\
\hline & NHS Great Yarmouth \& Waveney & 214,800 & 0.78 & 0.80 & 0.93 & 0.98 & 1.01 & 0.84 & 1.21 & 531 & 2.7 \\
\hline & NHS Ipswich and East Suffolk* & 399,500 & 0.88 & 0.84 & 0.90 & 0.88 & 0.91 & 0.79 & 1.05 & 478 & 5.6 \\
\hline & NHS North Norfolk & 170,600 & 0.93 & 0.82 & 1.01 & 0.91 & 0.93 & 0.75 & 1.14 & 522 & 1.5 \\
\hline & NHS Norwich & 198,200 & 0.77 & 0.72 & 0.89 & 0.90 & 0.93 & 0.76 & 1.15 & 444 & 7.3 \\
\hline & NHS South Norfolk* & 243,400 & 0.85 & 0.84 & 0.98 & 0.95 & 0.98 & 0.82 & 1.16 & 518 & 2.6 \\
\hline & NHS West Norfolk* & 174,100 & 0.82 & 0.86 & 0.83 & 0.85 & & & & & 2.6 \\
\hline & NHS West Suffolk* & 226,300 & 0.92 & 0.95 & 0.91 & 0.87 & & & & & 4.6 \\
\hline \multirow[t]{7}{*}{ Essex } & NHS Basildon and Brentwood & 257,800 & 0.95 & 0.92 & 1.03 & 0.91 & 0.89 & 0.74 & 1.06 & 442 & 7.1 \\
\hline & NHS Castle Point, Rayleigh and Rochford & 174,300 & 0.85 & 0.82 & 0.88 & 0.98 & 0.90 & 0.72 & 1.11 & 482 & 3.0 \\
\hline & NHS Mid Essex* & 385,700 & 1.00 & 0.92 & 0.98 & 0.94 & 0.93 & 0.80 & 1.07 & 485 & 4.4 \\
\hline & NHS North East Essex* & 325,100 & 0.94 & 0.92 & 0.94 & 0.99 & 0.95 & 0.81 & 1.11 & 483 & 5.5 \\
\hline & NHS Southend & 178,700 & 0.83 & 0.89 & 0.96 & 0.97 & 0.92 & 0.74 & 1.14 & 464 & 8.4 \\
\hline & NHS Thurrock & 165,200 & 0.86 & 0.83 & 0.81 & 0.81 & 0.80 & 0.62 & 1.02 & 381 & 14.1 \\
\hline & NHS West Essex* & 300,200 & 0.86 & 0.89 & 0.85 & 0.88 & 0.87 & 0.73 & 1.03 & 440 & 8.2 \\
\hline \multirow{7}{*}{$\begin{array}{l}\text { Hertfordshire } \\
\text { and the } \\
\text { South } \\
\text { Midlands }\end{array}$} & NHS Bedfordshire* & 440,300 & 1.00 & 1.08 & 1.04 & 1.06 & 1.01 & 0.88 & 1.15 & 513 & 11.2 \\
\hline & NHS Corby & 66,900 & 0.87 & 0.80 & 0.74 & 0.68 & 0.89 & 0.62 & 1.28 & 434 & 4.5 \\
\hline & NHS East and North Hertfordshire* & 559,100 & 0.94 & 0.97 & 0.97 & 0.99 & 0.97 & 0.86 & 1.09 & 478 & 10.4 \\
\hline & NHS Herts Valleys & 588,200 & 1.00 & 0.98 & 0.99 & 1.01 & 1.03 & 0.92 & 1.16 & 508 & 14.6 \\
\hline & NHS Luton* & 214,700 & 1.14 & 1.22 & 1.24 & 1.33 & 1.42 & 1.20 & 1.68 & 619 & 45.3 \\
\hline & NHS Milton Keynes & 267,800 & 1.02 & 1.04 & 0.98 & 1.06 & 1.11 & 0.94 & 1.30 & 534 & 19.6 \\
\hline & NHS Nene & 640,000 & 0.97 & 0.91 & 0.90 & 0.95 & 0.95 & 0.85 & 1.06 & 483 & 9.1 \\
\hline \multirow{7}{*}{$\begin{array}{l}\text { Leicestershire } \\
\text { and } \\
\text { Lincolnshire }\end{array}$} & NHS East Leicestershire and Rutland & 325,900 & 0.91 & 0.89 & 0.88 & 0.92 & 0.90 & 0.77 & 1.05 & 476 & 9.8 \\
\hline & NHS Leicester City & 342,600 & 1.49 & 1.49 & 1.54 & 1.62 & 1.64 & 1.44 & 1.86 & 706 & 49.5 \\
\hline & NHS Lincolnshire East & 232,000 & 0.85 & 0.84 & 0.85 & 0.85 & 0.84 & 0.70 & 1.02 & 465 & 2.0 \\
\hline & NHS Lincolnshire West & 234,300 & 0.84 & 0.81 & 0.83 & 0.83 & 0.76 & 0.62 & 0.93 & 384 & 3.0 \\
\hline & NHS South Lincolnshire* & 146,000 & 0.65 & 0.67 & 0.63 & 0.70 & 0.69 & 0.53 & 0.90 & 370 & 2.3 \\
\hline & NHS South West Lincolnshire & 124,300 & 0.74 & 0.73 & 0.71 & 0.71 & 0.69 & 0.51 & 0.92 & 370 & 2.3 \\
\hline & NHS West Leicestershire & 387,500 & 1.05 & 1.03 & 1.02 & 1.00 & 1.00 & 0.88 & 1.15 & 519 & 6.9 \\
\hline \multirow{8}{*}{$\begin{array}{l}\text { Shropshire } \\
\text { and } \\
\text { Staffordshire }\end{array}$} & NHS Cannock Chase & 134,900 & 0.76 & 0.72 & 0.74 & 0.70 & 0.65 & 0.49 & 0.87 & 341 & 2.4 \\
\hline & NHS East Staffordshire & 125,700 & 0.59 & 0.56 & 0.66 & 0.61 & 0.66 & 0.49 & 0.89 & 342 & 9.0 \\
\hline & NHS North Staffordshire & 216,700 & 0.90 & 0.92 & 0.94 & 0.88 & 0.90 & 0.74 & 1.09 & 480 & 3.5 \\
\hline & NHS Shropshire & 311,400 & 0.83 & 0.76 & 0.72 & 0.71 & 0.75 & 0.63 & 0.89 & 408 & 2.0 \\
\hline & NHS South East Staffs and Seisdon and Peninsular & 224,800 & 0.89 & 0.82 & 0.85 & 0.87 & 0.86 & 0.71 & 1.05 & 463 & 3.6 \\
\hline & NHS Stafford and Surrounds & 152,200 & 0.87 & 0.88 & 0.90 & 0.92 & 0.93 & 0.75 & 1.17 & 506 & 4.7 \\
\hline & NHS Stoke on Trent & 259,900 & 1.00 & 1.02 & 0.96 & 0.96 & 0.93 & 0.78 & 1.12 & 458 & 11.0 \\
\hline & NHS Telford \& Wrekin & 171,200 & 0.71 & 0.66 & 0.72 & 0.70 & 0.77 & 0.61 & 0.98 & 386 & 7.3 \\
\hline
\end{tabular}


Table 3.4. Continued

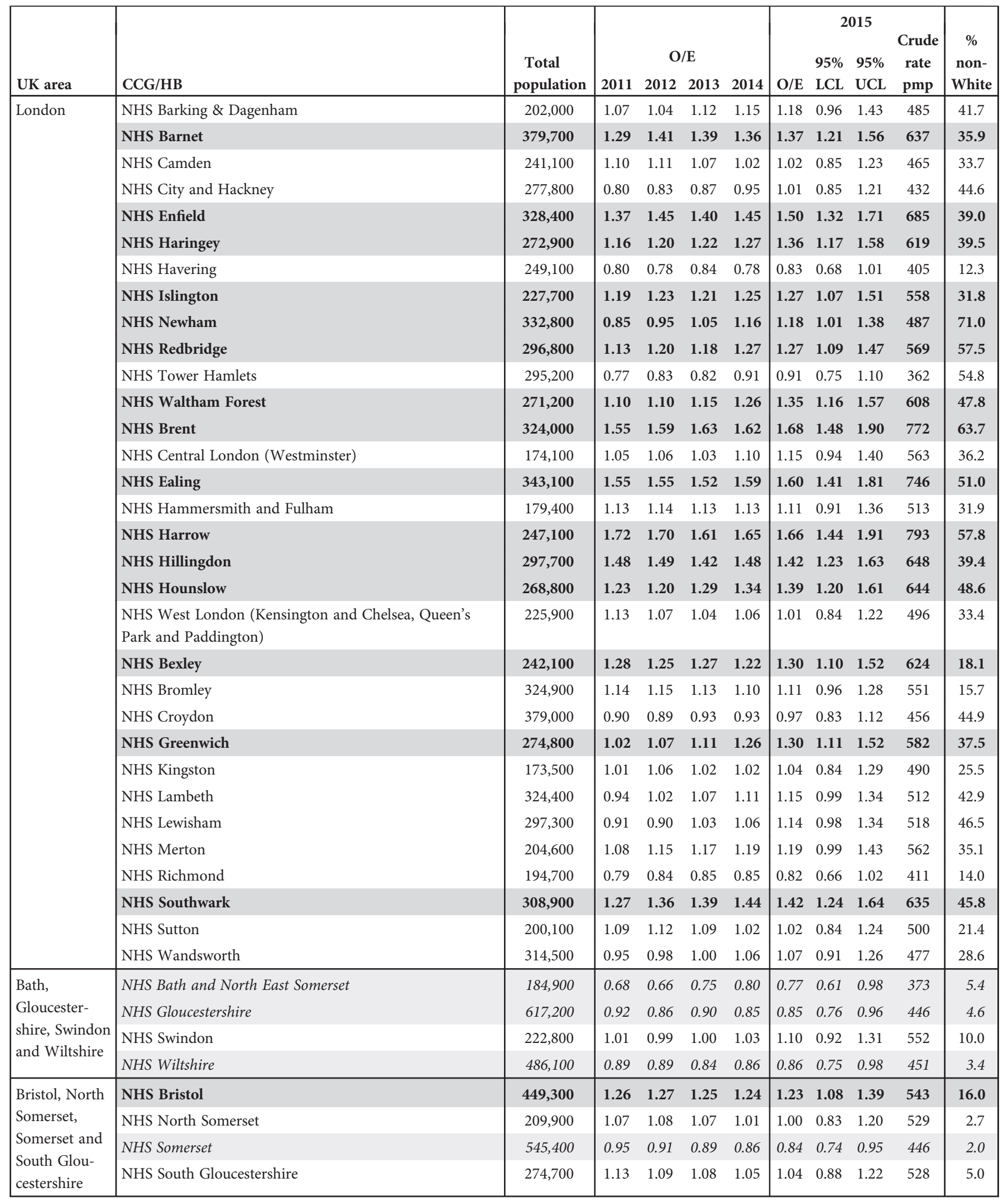


Table 3.4. Continued

\begin{tabular}{|c|c|c|c|c|c|c|c|c|c|c|c|}
\hline UK area & CCG/HB & $\begin{array}{c}\text { Total } \\
\text { population }\end{array}$ & \multicolumn{4}{|c|}{$\mathrm{O} / \mathrm{E}$} & $\mathrm{O} / \mathrm{E}$ & $\begin{array}{l}95 \% \\
\text { LCL } \\
\end{array}$ & $\begin{array}{l}95 \% \\
\text { UCL } \\
\end{array}$ & $\begin{array}{l}\text { Crude } \\
\text { rate } \\
\text { pmp } \\
\end{array}$ & $\begin{array}{c}\% \\
\text { non- } \\
\text { White }\end{array}$ \\
\hline \multirow{3}{*}{$\begin{array}{l}\text { Devon, } \\
\text { Cornwall and } \\
\text { Isles of Scilly }\end{array}$} & NHS Kernow & 551,700 & 1.13 & 1.16 & 1.12 & 1.09 & 1.08 & 0.97 & 1.21 & 584 & 1.8 \\
\hline & NHS North, East, West Devon & 890,600 & 1.04 & 1.04 & 1.04 & 1.01 & 1.00 & 0.91 & 1.09 & 513 & 3.0 \\
\hline & NHS South Devon and Torbay & 278,600 & 1.16 & 1.11 & 1.14 & 1.13 & 1.08 & 0.92 & 1.25 & 589 & 2.1 \\
\hline \multirow{8}{*}{$\begin{array}{l}\text { Kent and } \\
\text { Medway }\end{array}$} & NHS Ashford & 124,300 & 1.15 & 1.21 & 1.13 & 1.15 & 1.12 & 0.88 & 1.41 & 563 & 6.3 \\
\hline & NHS Canterbury and Coastal & 207,700 & 1.10 & 1.18 & 1.13 & 1.17 & 1.10 & 0.92 & 1.33 & 539 & 5.9 \\
\hline & NHS Dartford, Gravesham and Swanley & 258,200 & 1.18 & 1.16 & 1.15 & 1.18 & 1.21 & 1.03 & 1.41 & 596 & 13.0 \\
\hline & NHS Medway & 276,500 & 0.97 & 0.96 & 0.98 & 0.94 & 0.91 & 0.76 & 1.09 & 445 & 10.4 \\
\hline & NHS South Kent Coast & 205,500 & 0.84 & 0.85 & 0.86 & 0.92 & 0.90 & 0.74 & 1.10 & 482 & 4.5 \\
\hline & NHS Swale & 112,500 & 1.32 & 1.34 & 1.38 & 1.33 & 1.30 & 1.04 & 1.64 & 658 & 3.8 \\
\hline & NHS Thanet & 139,800 & 1.06 & 1.19 & 1.20 & 1.18 & 1.21 & 0.98 & 1.49 & 615 & 4.5 \\
\hline & NHS West Kent & 476,800 & 0.88 & 0.89 & 0.88 & 0.87 & 0.86 & 0.75 & 0.98 & 438 & 4.9 \\
\hline \multirow{12}{*}{$\begin{array}{l}\text { Surrey and } \\
\text { Sussex }\end{array}$} & NHS Brighton \& Hove & 285,300 & 0.90 & 0.88 & 0.84 & 0.84 & 0.88 & 0.73 & 1.05 & 414 & 10.9 \\
\hline & NHS Coastal West Sussex & 495,000 & 1.01 & 0.95 & 0.96 & 0.94 & 0.93 & 0.82 & 1.06 & 495 & 3.8 \\
\hline & NHS Crawley & 110,900 & 0.75 & 0.76 & 0.71 & 0.70 & 0.65 & 0.46 & 0.91 & 307 & 20.1 \\
\hline & NHS East Surrey & 182,000 & 0.83 & 0.80 & 0.80 & 0.74 & 0.75 & 0.59 & 0.94 & 379 & 8.3 \\
\hline & NHS Eastbourne, Hailsham and Seaford & 188,100 & 0.77 & 0.75 & 0.75 & 0.73 & 0.73 & 0.57 & 0.92 & 377 & 4.4 \\
\hline & NHS Guildford and Waverley & 206,100 & 0.65 & 0.71 & 0.70 & 0.71 & 0.70 & 0.56 & 0.89 & 344 & 7.2 \\
\hline & NHS Hastings \& Rother & 184,400 & 0.84 & 0.78 & 0.77 & 0.79 & 0.76 & 0.61 & 0.95 & 407 & 4.6 \\
\hline & NHS High Weald Lewes Havens & 171,600 & 0.76 & 0.85 & 0.79 & 0.79 & 0.73 & 0.58 & 0.93 & 396 & 3.1 \\
\hline & NHS Horsham and Mid Sussex & 230,300 & 0.74 & 0.72 & 0.73 & 0.80 & 0.82 & 0.67 & 1.00 & 425 & 4.9 \\
\hline & NHS North West Surrey & 343,000 & 1.02 & 1.02 & 1.00 & 0.98 & 0.97 & 0.83 & 1.12 & 490 & 12.5 \\
\hline & NHS Surrey Downs & 287,000 & 0.96 & 0.91 & 0.91 & 0.91 & 0.91 & 0.77 & 1.08 & 474 & 9.1 \\
\hline & NHS Surrey Heath & 95,900 & 1.20 & 1.21 & 1.06 & 0.93 & 0.90 & 0.67 & 1.21 & 469 & 9.3 \\
\hline \multirow{10}{*}{$\begin{array}{l}\text { Thames } \\
\text { Valley }\end{array}$} & NHS Aylesbury Vale & 207,000 & 1.22 & 1.22 & 1.16 & 1.13 & 1.10 & 0.91 & 1.31 & 560 & 9.7 \\
\hline & NHS Bracknell and Ascot & 137,000 & 1.08 & 1.06 & 1.05 & 0.99 & 0.95 & 0.74 & 1.21 & 474 & 9.5 \\
\hline & NHS Chiltern & 324,000 & 1.00 & 1.06 & 1.04 & 0.99 & 0.99 & 0.85 & 1.16 & 503 & 15.8 \\
\hline & NHS Newbury and District & 106,400 & 1.33 & 1.29 & 1.22 & 1.14 & 1.04 & 0.80 & 1.34 & 536 & 4.4 \\
\hline & NHS North \& West Reading & 100,300 & 0.99 & 1.01 & 1.05 & 1.00 & 0.95 & 0.72 & 1.26 & 488 & 10.4 \\
\hline & NHS Oxfordshire & 663,600 & 1.06 & 1.08 & 1.05 & 1.08 & 1.08 & 0.97 & 1.20 & 530 & 9.3 \\
\hline & NHS Slough & 145,700 & 1.62 & 1.63 & 1.83 & 1.84 & 1.93 & 1.61 & 2.30 & 844 & 54.3 \\
\hline & NHS South Reading & 111,000 & 1.39 & 1.29 & 1.31 & 1.36 & 1.46 & 1.15 & 1.85 & 621 & 30.5 \\
\hline & NHS Windsor, Ascot and Maidenhead & 141,400 & 1.08 & 1.21 & 1.25 & 1.29 & 1.26 & 1.02 & 1.55 & 623 & 14.7 \\
\hline & NHS Wokingham & 160,400 & 1.00 & 1.00 & 0.97 & 0.98 & 0.97 & 0.78 & 1.21 & 499 & 11.6 \\
\hline \multirow[t]{9}{*}{ Wessex } & NHS Dorset & 765,700 & 0.96 & 0.90 & 0.86 & 0.86 & 0.85 & 0.76 & 0.94 & 445 & 4.0 \\
\hline & NHS Fareham and Gosport & 199,500 & 0.99 & 0.93 & 1.02 & 1.01 & 0.99 & 0.81 & 1.20 & 516 & 3.4 \\
\hline & NHS Isle of Wight & 139,400 & 0.77 & 0.74 & 0.64 & 0.63 & 0.69 & 0.53 & 0.90 & 380 & 2.7 \\
\hline & NHS North East Hampshire and Farnham & 209,200 & 0.84 & 0.85 & 0.86 & 0.88 & 0.89 & 0.73 & 1.09 & 449 & 9.7 \\
\hline & NHS North Hampshire & 220,800 & 0.86 & 0.86 & 0.83 & 0.82 & 0.85 & 0.70 & 1.04 & 439 & 6.4 \\
\hline & NHS Portsmouth & 211,800 & 1.01 & 0.98 & 0.98 & 0.92 & 0.87 & 0.71 & 1.08 & 397 & 11.6 \\
\hline & NHS South Eastern Hampshire & 211,900 & 0.98 & 1.00 & 0.96 & 1.02 & 1.00 & 0.83 & 1.21 & 529 & 3.1 \\
\hline & NHS Southampton & 249,500 & 1.02 & 1.06 & 1.08 & 1.10 & 1.10 & 0.92 & 1.32 & 485 & 14.1 \\
\hline & NHS West Hampshire & 554,900 & 0.99 & 0.95 & 0.92 & 0.90 & 0.87 & 0.77 & 0.99 & 460 & 3.9 \\
\hline \multirow[t]{7}{*}{ Wales } & Betsi Cadwaladr University & 694,500 & 0.88 & 0.82 & 0.73 & 0.72 & 0.85 & 0.76 & 0.95 & 446 & 2.5 \\
\hline & Powys Teaching & 132,600 & 0.95 & 0.80 & 0.78 & 0.76 & 0.73 & 0.56 & 0.96 & 407 & 1.6 \\
\hline & Hywel Dda & 383,200 & 1.08 & 1.00 & 1.06 & 1.00 & 0.95 & 0.83 & 1.10 & 504 & 2.2 \\
\hline & Abertawe Bro Morgannwg University & 525,500 & 1.32 & 1.32 & 1.29 & 1.25 & 1.20 & 1.08 & 1.34 & 613 & 3.9 \\
\hline & Cwm Taf & 296,700 & 1.62 & 1.59 & 1.60 & 1.50 & 1.43 & 1.25 & 1.63 & 721 & 2.6 \\
\hline & Aneurin Bevan & 581,800 & 1.27 & 1.34 & 1.28 & 1.23 & 1.18 & 1.07 & 1.31 & 608 & 3.9 \\
\hline & Cardiff and Vale University & 484,800 & 1.22 & 1.24 & 1.19 & 1.13 & 1.14 & 1.01 & 1.28 & 532 & 12.2 \\
\hline
\end{tabular}


Table 3.4. Continued

\begin{tabular}{|c|c|c|c|c|c|c|c|c|c|c|c|}
\hline \multirow[b]{3}{*}{ UK area } & \multirow[b]{3}{*}{ CCG/HB } & \multirow{3}{*}{$\begin{array}{c}\text { Total } \\
\text { population }\end{array}$} & \multirow[b]{3}{*}{2011} & \multirow{2}{*}{\multicolumn{3}{|c|}{$\mathrm{O} / \mathrm{E}$}} & \multicolumn{3}{|c|}{2015} & \multirow{3}{*}{$\begin{array}{c}\text { Crude } \\
\text { rate } \\
\text { pmp }\end{array}$} & \multirow{3}{*}{$\begin{array}{c}\% \\
\text { non- } \\
\text { White }\end{array}$} \\
\hline & & & & & & & & $95 \%$ & $95 \%$ & & \\
\hline & & & & 2012 & 2013 & 2014 & $\mathrm{O} / \mathrm{E}$ & LCL & UCL & & \\
\hline \multirow[t]{14}{*}{ Scotland } & Ayrshire and Arran & 370,600 & 0.95 & 0.96 & 0.96 & 0.97 & 0.96 & 0.84 & 1.11 & 521 & 1.2 \\
\hline & Borders & 114,000 & 1.03 & 1.10 & 1.04 & 1.00 & 0.95 & 0.74 & 1.22 & 535 & 1.3 \\
\hline & Dumfries and Galloway & 149,700 & 0.93 & 0.88 & 0.82 & 0.84 & 0.86 & 0.68 & 1.09 & 481 & 1.2 \\
\hline & Fife & 368,100 & 0.87 & 0.87 & 0.87 & 0.85 & 0.84 & 0.72 & 0.98 & 443 & 2.4 \\
\hline & Forth Valley & 302,700 & 0.85 & 0.87 & 0.87 & 0.92 & 0.93 & 0.79 & 1.09 & 489 & 2.2 \\
\hline & Grampian & 587,800 & 0.91 & 0.91 & 0.92 & 0.88 & 0.91 & 0.81 & 1.02 & 468 & 4.0 \\
\hline & Greater Glasgow and Clyde & $1,149,900$ & 1.09 & 1.15 & 1.16 & 1.16 & 1.14 & 1.06 & 1.23 & 576 & 7.3 \\
\hline & Highland & 321,000 & 1.11 & 1.05 & 1.03 & 1.02 & 1.02 & 0.88 & 1.18 & 564 & 1.3 \\
\hline & Lanarkshire & 654,500 & 1.04 & 1.06 & 1.03 & 1.07 & 1.04 & 0.94 & 1.16 & 547 & 2.0 \\
\hline & Lothian & 867,800 & 0.90 & 0.87 & 0.84 & 0.85 & 0.84 & 0.76 & 0.93 & 419 & 5.6 \\
\hline & Orkney & 21,700 & 0.84 & 0.79 & 0.74 & 0.52 & 0.50 & 0.22 & 1.10 & 277 & 0.7 \\
\hline & Shetland & 23,200 & 0.51 & 0.58 & 0.54 & 0.51 & 0.48 & 0.22 & 1.08 & 259 & 1.5 \\
\hline & Tayside & 415,000 & 1.00 & 0.96 & 0.94 & 0.90 & 0.90 & 0.78 & 1.03 & 465 & 3.2 \\
\hline & Western Isles & 27,100 & 0.75 & 0.71 & 0.66 & 0.62 & 0.59 & 0.31 & 1.13 & 332 & 0.9 \\
\hline \multirow{5}{*}{$\begin{array}{l}\text { Northern } \\
\text { Ireland }\end{array}$} & Belfast & 353,800 & 1.10 & 1.14 & 1.13 & 1.18 & 1.19 & 1.04 & 1.37 & 557 & 3.2 \\
\hline & Northern & 471,200 & 0.95 & 0.93 & 0.94 & 0.99 & 1.01 & 0.89 & 1.15 & 501 & 1.2 \\
\hline & Southern & 373,000 & 0.89 & 0.96 & 0.96 & 1.02 & 1.12 & 0.97 & 1.29 & 528 & 1.2 \\
\hline & South Eastern & 354,700 & 0.96 & 0.92 & 0.92 & 0.96 & 1.00 & 0.87 & 1.16 & 505 & 1.3 \\
\hline & Western & 299,000 & 0.92 & 0.89 & 1.02 & 1.15 & 1.20 & 1.03 & 1.39 & 582 & 1.0 \\
\hline
\end{tabular}

The prevalence of renal transplant recipients in each CCG in England, Northern Ireland (Health and Social Care Trust Areas), Scotland (Health Boards) and Wales (Local Health Boards) and the proportion of prevalent patients according to modality in the renal centres across the UK are described in tables 3.4 and 3.5 respectively.

After standardisation for age and gender, unexplained variability was evident in the prevalence of renal transplant recipients, with some areas having higher than the predicted number of prevalent transplant patients per million population and others lower. There are a number of potential explanations for these inconsistencies, including geographical differences in access to renal transplantation in the UK. This has previously been analysed in detail by the UKRR [2] and is currently the focus of a large national study (access to Transplant and Transplant Outcome Measures (ATTOM)) [3].

The proportion of prevalent RRT patients with a transplant relative to the number on dialysis has gradually risen over the last decade.

\section{Age and gender}

The gender ratio amongst incident and prevalent kidney transplant patients has remained stable for at least the last ten years (table 3.6, figure 3.1). The median age of incident transplant recipients has increased during the same time period, which reflects changes to the renal replacement therapy population. This is mirrored by an increase in the median age of the prevalent population, which reflects the increase in age at which patients are transplanted, the increase access to transplantation for older recipients, as well as improved survival after kidney transplantation over the last 10 years.

\section{Primary renal diagnosis}

The primary renal diagnosis of patients receiving a kidney transplant in the UK has remained relatively stable over the last five years (table 3.7).

\section{Ethnicity}

The ethnicity of those receiving a kidney transplant between 2010 and 2015 is shown in table 3.8. A comparison of the proportion of patients within each ethnic group receiving a transplant to those commencing dialysis from the same group is difficult because data on ethnicity were missing, or there was a high proportion with ethnicity classified as 'missing'. This is a particular issue in Scotland, where ethnicity reporting is not mandatory. Analysis isolated to the remainder of the 
Table 3.5. Distribution of prevalent patients on RRT by centre and modality on 31/12/2015

\begin{tabular}{|c|c|c|c|c|}
\hline Centre & $N$ & $\% \mathrm{HD}$ & $\% \mathrm{PD}$ & $\%$ transplant \\
\hline \multicolumn{5}{|c|}{ Transplant centres } \\
\hline B QEH & 2,254 & 45 & 6 & 49 \\
\hline Bristol & 1,477 & 36 & 4 & 61 \\
\hline $\mathrm{Camb}^{\mathrm{a}}$ & 1,539 & 38 & 3 & 59 \\
\hline Cardff & 1,613 & 31 & 5 & 64 \\
\hline Edinb & 773 & 37 & 3 & 60 \\
\hline Glasgw & 1,715 & 35 & 3 & 62 \\
\hline L Barts & 2,286 & 44 & 9 & 47 \\
\hline L Guys & 2,011 & 34 & 2 & 65 \\
\hline L Rfree & 2,088 & 34 & 7 & 58 \\
\hline L St.G ${ }^{\mathrm{b}}$ & 845 & 40 & 6 & 54 \\
\hline $\mathrm{M} \mathrm{RI}^{\mathrm{b}}$ & 1,896 & 28 & 3 & 69 \\
\hline Newc & 1,010 & 31 & 5 & 64 \\
\hline Nottm & 1,114 & 35 & 7 & 58 \\
\hline Oxford $^{\mathrm{b}}$ & 1,697 & 26 & 6 & 69 \\
\hline Plymth & 505 & 27 & 7 & 66 \\
\hline Ports & 1,671 & 40 & 4 & 56 \\
\hline Sheff $^{\text {b }}$ & 1,390 & 43 & 5 & 53 \\
\hline \multicolumn{5}{|c|}{ Dialysis centres } \\
\hline Abrdn & 532 & 41 & 5 & 54 \\
\hline Airdrie & 425 & 46 & 4 & 50 \\
\hline Antrim & 239 & 51 & 8 & 41 \\
\hline Chelms & 285 & 51 & 9 & 40 \\
\hline Clwyd & 185 & 45 & 11 & 44 \\
\hline Colchr & 120 & 100 & & \\
\hline D \& Gall & 130 & 42 & 8 & 50 \\
\hline Derby $^{b}$ & 537 & 45 & 15 & 40 \\
\hline Donc & 301 & 60 & 8 & 32 \\
\hline Dorset & 679 & 43 & 6 & 51 \\
\hline Dudley & 312 & 55 & 18 & 27 \\
\hline Dundee & 421 & 44 & 4 & 52 \\
\hline Exeter ${ }^{\mathrm{b}}$ & 965 & 45 & 9 & 46 \\
\hline Glouc & 443 & 51 & 8 & 40 \\
\hline Hull $^{\mathrm{b}}$ & 857 & 42 & 9 & 49 \\
\hline Inverns & 253 & 37 & 5 & 58 \\
\hline Ipswi & 407 & 35 & 9 & 56 \\
\hline Kent & 1,042 & 41 & 6 & 54 \\
\hline Klmarnk & 309 & 44 & 12 & 44 \\
\hline Krkcldy & 295 & 51 & 7 & 42 \\
\hline L Kings & 1,085 & 52 & 8 & 40 \\
\hline
\end{tabular}


Table 3.5. Continued

\begin{tabular}{|c|c|c|c|c|}
\hline Centre & $N$ & $\% \mathrm{HD}$ & $\% \mathrm{PD}$ & $\%$ transplant \\
\hline Liv Ain & 228 & 77 & 17 & 7 \\
\hline Middlbr ${ }^{b}$ & 902 & 39 & 2 & 58 \\
\hline Norwch & 741 & 46 & 5 & 49 \\
\hline Prestn $^{\mathrm{b}}$ & 1,217 & 47 & 4 & 49 \\
\hline Redng & 778 & 39 & 8 & 53 \\
\hline Shrew & 370 & 55 & 9 & 36 \\
\hline Stevng & 827 & 62 & 2 & 37 \\
\hline Sthend & 246 & 51 & 7 & 42 \\
\hline Stoke & 789 & 42 & 10 & 48 \\
\hline Sund & 459 & 48 & 4 & 48 \\
\hline Swanse ${ }^{b}$ & 757 & 48 & 8 & 44 \\
\hline Wolve & 581 & 55 & 14 & 32 \\
\hline Wrexm & 293 & 38 & 13 & 49 \\
\hline York & 489 & 33 & 6 & 61 \\
\hline England & 51,672 & 41 & 6 & 53 \\
\hline N Ireland & 1,701 & 37 & 5 & 58 \\
\hline Scotland & 4,853 & 40 & 5 & 56 \\
\hline Wales & 3,030 & 38 & 7 & 55 \\
\hline UK & 61,256 & 41 & 6 & 53 \\
\hline
\end{tabular}

${ }^{\mathrm{a}}$ Cambridge was unable to submit any patient level data for 2015 but provided the total number of adult patients on treatment at the end of the year by treatment modality. Those numbers have been added in tables 3.3 and 3.5 only, therefore Cambridge is not included in any of the centre level analyses

${ }^{\mathrm{b}}$ Subsequent to closing the 2015 database some centres reported variation to the numbers returned for 2015 . Tables 3.3 and 3.5 (but not the reminder of this chapter) reflect these revisions. For most centres the change reported was small ( $<5$ patients), but a few centres reported notable numbers of patients not submitted (Sheffield 51 HD, 6 PD, 8 transplant; Salford 2 HD, 9 PD, 2 transplant and Middlesbrough 9 transplant patients)

Blank cells: no patients on that modality

Table 3.6. Median age and gender ratio of incident and prevalent transplant patients 2010-2015

\begin{tabular}{|c|c|c|c|c|c|c|}
\hline \multirow[b]{2}{*}{ Year } & \multicolumn{3}{|c|}{ Incident transplants } & \multicolumn{3}{|c|}{ Prevalent transplants* } \\
\hline & $N$ & Median age & $\mathrm{M}: \mathrm{F}$ ratio & $N$ & Median age & $M: F$ ratio \\
\hline 2010 & 2,584 & 49.6 & 1.7 & 24,885 & 51.2 & 1.5 \\
\hline 2011 & 2,628 & 49.1 & 1.7 & 26,172 & 51.7 & 1.6 \\
\hline 2012 & 2,782 & 50.5 & 1.6 & 27,535 & 52.3 & 1.5 \\
\hline 2013 & 3,128 & 50.4 & 1.6 & 29,442 & 52.8 & 1.6 \\
\hline 2014 & 3,031 & 50.6 & 1.5 & 31,044 & 53.3 & 1.5 \\
\hline 2015 & 2,864 & 50.9 & 1.5 & 31,692 & 53.8 & 1.5 \\
\hline
\end{tabular}

*As on 31 st December for given year 


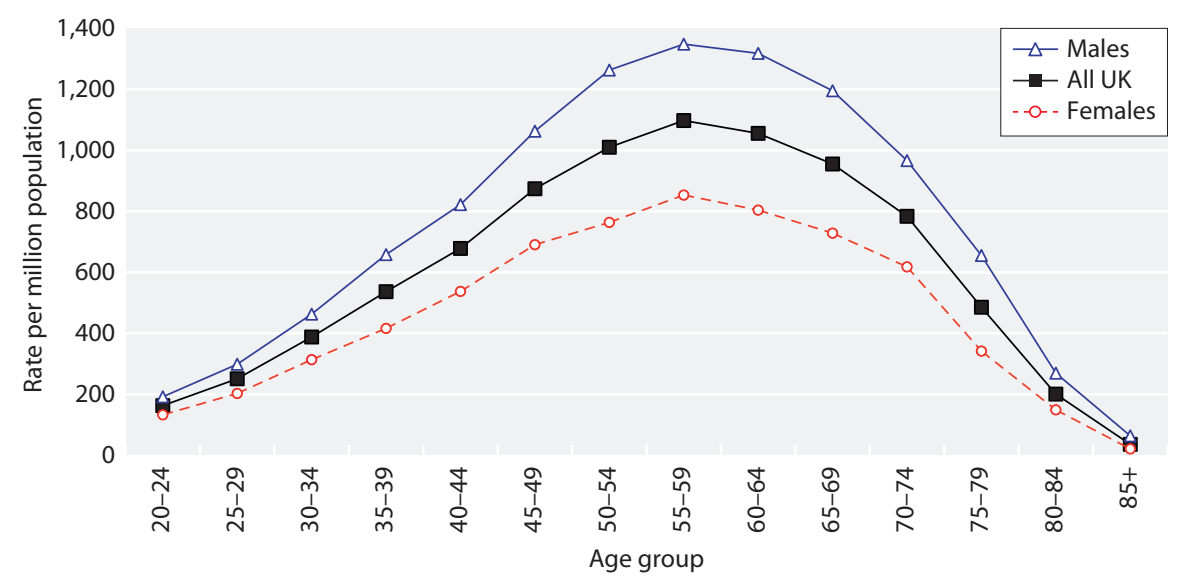

Fig. 3.1. Transplant prevalence rate per million population by age and gender on $31 / 12 / 2015$

Table 3.7. Primary renal diagnosis in renal transplant recipients 2010-2015

\begin{tabular}{|c|c|c|c|c|c|c|c|c|c|}
\hline \multirow{2}{*}{ Primary renal diagnosis } & \multicolumn{7}{|c|}{ New transplants by year } & \multicolumn{2}{|c|}{$\begin{array}{l}\text { Established transplants } \\
\text { on } 31 / 12 / 2015\end{array}$} \\
\hline & 2010 & 2011 & 2012 & 2013 & 2014 & & & & \\
\hline Aetiology uncertain & 15.1 & 15.1 & 12.3 & 13.3 & 12.3 & 12.3 & 349 & 14.7 & 4,671 \\
\hline Diabetes & 13.1 & 13.6 & 15.1 & 13.9 & 15.3 & 15.1 & 430 & 10.6 & 3,375 \\
\hline Pyelonephritis & 10.7 & 10.2 & 10.5 & 10.2 & 9.0 & 9.2 & 261 & 12.8 & 4,054 \\
\hline Reno-vascular disease & 7.7 & 7.2 & 7.2 & 8.2 & 7.7 & 8.1 & 230 & 6.3 & 2,002 \\
\hline Other & 17.1 & 17.0 & 17.1 & 15.0 & 17.0 & 15.8 & 448 & 17.5 & 5,554 \\
\hline Not available & 1.2 & 1.0 & 1.2 & 2.6 & 3.0 & 4.4 & 124 & 1.4 & 447 \\
\hline
\end{tabular}

Table 3.8. Ethnicity of patients who received a transplant in the years 2010-2015

\begin{tabular}{|c|c|c|c|c|c|}
\hline 2010 & 76.9 & 10.5 & 5.7 & 2.6 & 4.3 \\
\hline 2011 & 76.3 & 9.6 & 6.2 & 2.9 & 5.1 \\
\hline 2013 & 71.5 & 12.1 & 6.9 & 2.8 & 6.7 \\
\hline 2014 & 69.2 & 12.3 & 6.5 & 4.2 & 7.8 \\
\hline 2015 & 67.5 & 12.7 & 7.4 & 3.9 & 8.4 \\
\hline
\end{tabular}

UK, where completeness of data was good, may allow assessment of variation in access to transplantation in future reports.

There has been a year on year increase in the percentage of incident kidney recipients from non-White ethnic groups, which reflects the changing population of the UK, the different incidence of CKD in different groups and improved access to transplantation across these ethnic backgrounds.

Outcomes in UK renal transplant recipients in 2015

\section{Clinical and laboratory outcomes}

\section{Introduction}

There continued to be marked variation in the completeness of data (tables 3.9a, 3.9b) reported by each renal centre, particularly for blood pressure and parathyroid hormone, which limits the ability to perform more meaningful comparisons between centres, or determine the causes of inter-centre differences in outcomes. 
Table 3.9a. Percentage completeness of ethnicity, eGFR and blood pressure by centre for prevalent transplant patients on $31 / 12 / 2015$

\begin{tabular}{|c|c|c|c|c|c|c|c|c|c|}
\hline Centre & $N$ & Ethnicity $^{\mathrm{a}}$ & eGFR & $\begin{array}{c}\text { Blood } \\
\text { pressure }^{b}\end{array}$ & Centre & $N$ & Ethnicity $^{\mathrm{a}}$ & eGFR & $\begin{array}{c}\text { Blood } \\
\text { pressure }^{\mathrm{b}}\end{array}$ \\
\hline \multicolumn{10}{|l|}{ England } \\
\hline B Heart & 180 & 100 & 94 & 0 & Salford & 479 & 100 & 97 & 0 \\
\hline B QEH & 1,057 & 100 & 95 & 0 & Sheff & 705 & 100 & 99 & 96 \\
\hline Basldn & 77 & 100 & 94 & 91 & Shrew & 134 & 100 & 98 & 0 \\
\hline Bradfd & 315 & 100 & 96 & 62 & Stevng & 286 & 100 & 98 & 45 \\
\hline Brightn & 436 & 99 & 98 & 49 & Sthend & 101 & 100 & 99 & 80 \\
\hline Bristol & 868 & 100 & 99 & 78 & Stoke & 368 & 100 & 99 & 0 \\
\hline $\mathrm{Camb}^{\mathrm{c}}$ & & & & & Sund & 218 & 100 & 99 & 0 \\
\hline Carlis & 158 & 100 & 92 & 0 & Truro & 222 & 100 & 99 & 3 \\
\hline Carsh & 640 & 100 & 91 & 4 & Wirral & 19 & 100 & 84 & 0 \\
\hline Chelms & 112 & 98 & 96 & 96 & Wolve & 184 & 100 & 96 & 73 \\
\hline Covnt & 504 & 100 & 97 & 87 & York & 294 & 99 & 95 & 69 \\
\hline Derby & 200 & 100 & 98 & 92 & N Ireland & & & & \\
\hline Donc & 82 & 100 & 99 & 98 & Antrim & 97 & 100 & 99 & 96 \\
\hline Dorset & 341 & 100 & 89 & 87 & Belfast & 543 & 99 & 100 & 52 \\
\hline Dudley & 80 & 100 & 96 & 40 & Newry & 104 & 100 & 98 & 88 \\
\hline Exeter & 430 & 100 & 99 & 90 & Ulster & 55 & 100 & 98 & 98 \\
\hline Glouc & 174 & 99 & 98 & $\begin{array}{r}80 \\
2\end{array}$ & West NI & 149 & 100 & 100 & 95 \\
\hline $\begin{array}{l}\text { Hull } \\
\text { Ipswi }\end{array}$ & $\begin{array}{l}411 \\
220\end{array}$ & $\begin{array}{l}99 \\
99\end{array}$ & $\begin{array}{l}91 \\
98\end{array}$ & $\begin{array}{r}2 \\
95\end{array}$ & Scotland & & & & \\
\hline Kent & 542 & 100 & 97 & 86 & Abrdn & 278 & 56 & 99 & $\mathrm{n} / \mathrm{a}$ \\
\hline L Barts & 1,028 & 100 & 68 & 0 & Airdrie & 212 & 61 & 73 & $\mathrm{n} / \mathrm{a}$ \\
\hline L Guys & 1,261 & 99 & 98 & 0 & D \& Gall & 65 & 28 & 88 & $\mathrm{n} / \mathrm{a}$ \\
\hline L Kings & 415 & 100 & 99 & 100 & Dundee & 211 & 62 & 98 & $\mathrm{n} / \mathrm{a}$ \\
\hline L RFree & 1,183 & 99 & 97 & 77 & Edinb & 451 & 25 & 94 & $\mathrm{n} / \mathrm{a}$ \\
\hline L St.G & 440 & 96 & 96 & 49 & Glasgw & 1,018 & 24 & 74 & $\mathrm{n} / \mathrm{a}$ \\
\hline L West & 1,762 & 100 & 98 & 0 & Inverns & 140 & 83 & 84 & $\mathrm{n} / \mathrm{a}$ \\
\hline Leeds & 918 & 100 & 99 & 98 & Klmarnk & 132 & 67 & 79 & $\mathrm{n} / \mathrm{a}$ \\
\hline Leic & 1,132 & 98 & 97 & 28 & Krkcldy & 119 & 36 & 97 & $\mathrm{n} / \mathrm{a}$ \\
\hline Liv Ain & 14 & 93 & 100 & 0 & Wales & & & & \\
\hline Liv Roy & 812 & 99 & 95 & 1 & Bangor & 81 & 100 & 99 & 83 \\
\hline M RI & 1,220 & 99 & 98 & 7 & Cardff & 1,006 & 100 & 99 & 97 \\
\hline Middlbr & 504 & 100 & 95 & 34 & Clwyd & 79 & 100 & 100 & 94 \\
\hline Newc & 630 & 100 & 98 & 96 & Swanse & 316 & 100 & 99 & 100 \\
\hline $\begin{array}{l}\text { Norwch } \\
\text { Nottm }\end{array}$ & $\begin{array}{l}352 \\
617\end{array}$ & $\begin{array}{l}100 \\
100\end{array}$ & $\begin{array}{r}99 \\
100\end{array}$ & $\begin{array}{r}2 \\
92\end{array}$ & Wrexm & 141 & 100 & 99 & 89 \\
\hline Oxford & 1,096 & 95 & 99 & 15 & England & 25,423 & 99 & 96 & 39 \\
\hline Plymth & 316 & 100 & 98 & 92 & $\mathbf{N}$ Ireland & 948 & 100 & 99 & 70 \\
\hline Ports & 909 & 99 & 95 & 11 & Scotland & 2,626 & 39 & 84 & $\mathbf{n} / \mathbf{a}$ \\
\hline Prestn & 576 & 100 & 98 & 0 & Wales & 1,623 & 100 & 99 & 96 \\
\hline Redng & 401 & 98 & 99 & 95 & UK & 30,620 & 94 & 95 & $43^{d}$ \\
\hline
\end{tabular}

n/a - not available

${ }^{a}$ Patients with missing ethnicity were classed as White for eGFR calculation

${ }^{\mathrm{b}} \mathrm{S}$ cottish centres excluded from blood pressure analysis as data not provided by the Scottish Renal Registry

${ }^{\mathrm{c}}$ Cambridge was unable to submit data for 2015

${ }^{\mathrm{d}}$ Excluding Scotland

The 71 renal centres in the UK comprise 52 centres in England, five in Wales, five in Northern Ireland and nine in Scotland. Colchester was reported as having no transplanted patients and was therefore excluded. Cambridge was unable to submit patient level data for 2015. After exclusion of these centres, prevalent patient data from 69 renal centres across the UK were analysed.

For the one-year post-transplant analyses, in which patients were assigned to the centre that performed their transplant, all 23 transplant centres across the UK were included in the analysis. 
Table 3.9b. Percentage completeness of haemoglobin, serum cholesterol, serum calcium, serum phosphate and serum PTH by centre for prevalent transplant patients on 31/12/2015

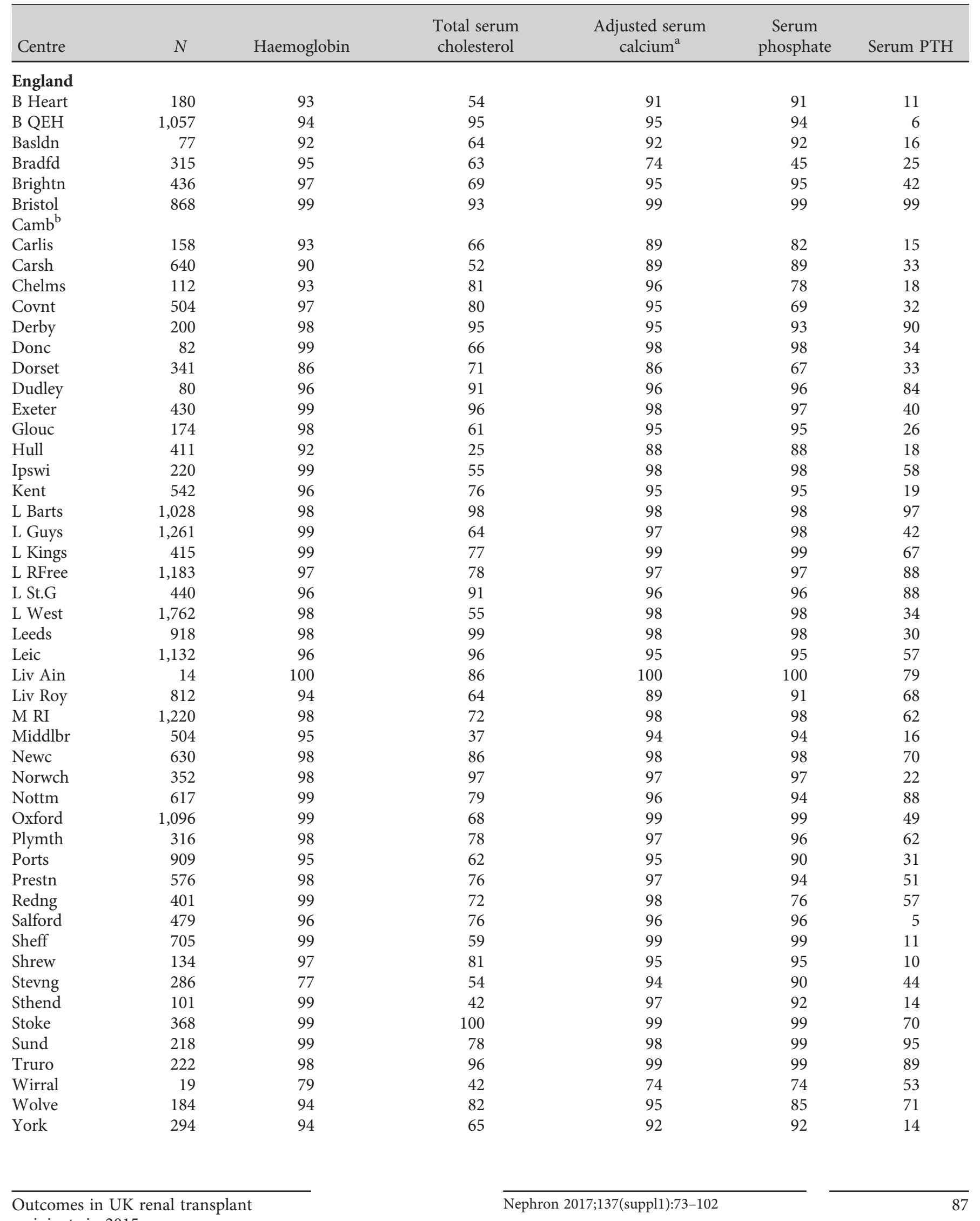


Table 3.9b. Continued

\begin{tabular}{|c|c|c|c|c|c|c|}
\hline Centre & $N$ & Haemoglobin & $\begin{array}{c}\text { Total serum } \\
\text { cholesterol }\end{array}$ & $\begin{array}{l}\text { Adjusted serum } \\
\text { calcium }^{\mathrm{a}}\end{array}$ & $\begin{array}{c}\text { Serum } \\
\text { phosphate }\end{array}$ & Serum PTH \\
\hline \multicolumn{7}{|l|}{ N Ireland } \\
\hline Antrim & 97 & 99 & 99 & 97 & 99 & 98 \\
\hline Belfast & 543 & 99 & 99 & 99 & 99 & 32 \\
\hline Newry & 104 & 97 & 99 & 96 & 97 & 97 \\
\hline Ulster & 55 & 98 & 100 & 95 & 98 & 15 \\
\hline West NI & 149 & 99 & 100 & 99 & 100 & 91 \\
\hline \multicolumn{7}{|l|}{ Scotland } \\
\hline Abrdn & 278 & 99 & $\mathrm{n} / \mathrm{a}$ & 96 & 96 & $\mathrm{n} / \mathrm{a}$ \\
\hline Airdrie & 212 & 98 & $\mathrm{n} / \mathrm{a}$ & 97 & 96 & $\mathrm{n} / \mathrm{a}$ \\
\hline D \& Gall & 65 & 98 & $\mathrm{n} / \mathrm{a}$ & 98 & 98 & $\mathrm{n} / \mathrm{a}$ \\
\hline Dundee & 211 & 98 & $\mathrm{n} / \mathrm{a}$ & 97 & 97 & $\mathrm{n} / \mathrm{a}$ \\
\hline Edinb & 451 & 94 & $\mathrm{n} / \mathrm{a}$ & 92 & 81 & $\mathrm{n} / \mathrm{a}$ \\
\hline Glasgw & 1,018 & 97 & $\mathrm{n} / \mathrm{a}$ & 97 & 97 & $\mathrm{n} / \mathrm{a}$ \\
\hline Inverns & 140 & 79 & $\mathrm{n} / \mathrm{a}$ & 72 & 68 & $\mathrm{n} / \mathrm{a}$ \\
\hline Klmarnk & 132 & 98 & $\mathrm{n} / \mathrm{a}$ & 97 & 96 & $\mathrm{n} / \mathrm{a}$ \\
\hline Krkcldy & 119 & 97 & $\mathrm{n} / \mathrm{a}$ & 97 & 97 & $\mathrm{n} / \mathrm{a}$ \\
\hline \multicolumn{7}{|l|}{ Wales } \\
\hline Bangor & 81 & 98 & 100 & 99 & 99 & 27 \\
\hline Cardff & 1,006 & 99 & 95 & 99 & 99 & 21 \\
\hline Clwyd & 79 & 96 & 100 & 99 & 99 & 81 \\
\hline Swanse & 316 & 99 & 91 & 99 & 99 & 72 \\
\hline Wrexm & 141 & 99 & 100 & 99 & 99 & 100 \\
\hline England & 25,423 & 97 & 75 & 96 & 94 & 49 \\
\hline N Ireland & 948 & 99 & 99 & 98 & 99 & 54 \\
\hline Scotland ${ }^{c}$ & 2,626 & 96 & $\mathbf{n} / \mathbf{a}$ & 95 & 93 & $\mathbf{n} / \mathbf{a}$ \\
\hline Wales & 1,623 & 99 & 95 & 99 & 99 & 41 \\
\hline UK & 30,620 & 97 & $77^{d}$ & 96 & 94 & $48^{d}$ \\
\hline
\end{tabular}

n/a - not available

${ }^{a}$ Serum calcium corrected for serum albumin

${ }^{\mathrm{b}}$ Cambridge was unable to submit data for 2015

${ }^{c}$ Dataset provided by the Scottish Renal Registry for Scottish centres shown did not include data on serum cholesterol or serum PTH

${ }^{\mathrm{d}}$ Excluding Scotland

\section{Methods}

Data for key laboratory variables are reported for all prevalent patients with valid data returns for a given renal centre (both transplanting and non-transplanting centres) and for one year post-transplant results for patients transplanted 2008-2014, with patients attributed to the transplant centre that performed the procedure.

Time since transplantation may have a significant effect on key biochemical and clinical variables and this is likely to be independent of a centre's clinical practices. Therefore, inter-centre comparison of data on prevalent transplant patients is open to bias. To minimise bias relating to fluctuations in biochemical and clinical parameters occurring in the initial post-transplant period, one year post-transplantation outcomes are also reported. It is presumed that patient selection policies and local clinical practices are more likely to be relevant in influencing outcomes 12 months post-transplant and therefore comparison of outcomes between centres is more robust. However, even the 12 months posttransplant comparisons could be biased by the fact that in some centres, repatriation of patients only occurs if the graft is failing whereas in others it only occurs if the graft function is stable.

Centres with $<10$ patients or $<50 \%$ data completeness have been excluded from the figures. Scottish centres were also excluded from blood pressure analyses as data were not provided.

\section{Prevalent patient data}

Biochemical and clinical data for patients with a functioning transplant followed in either a transplanting or non-transplanting centre were included in the analyses. The cohort consisted of prevalent patients as on 31st December 2015. Patients were considered as having a functioning transplant if 'transplant' was listed as the last mode of RRT in the last quarter of 2015. Patients were assigned to the renal centre that sent the data to the UKRR but some patients will have received care in more than one centre. If data for the same transplant patient were received from both the transplant centre and non-transplant centre, care was usually allocated to the non-transplant centre (see appendix B). Patients with a functioning transplant of less than three months duration 


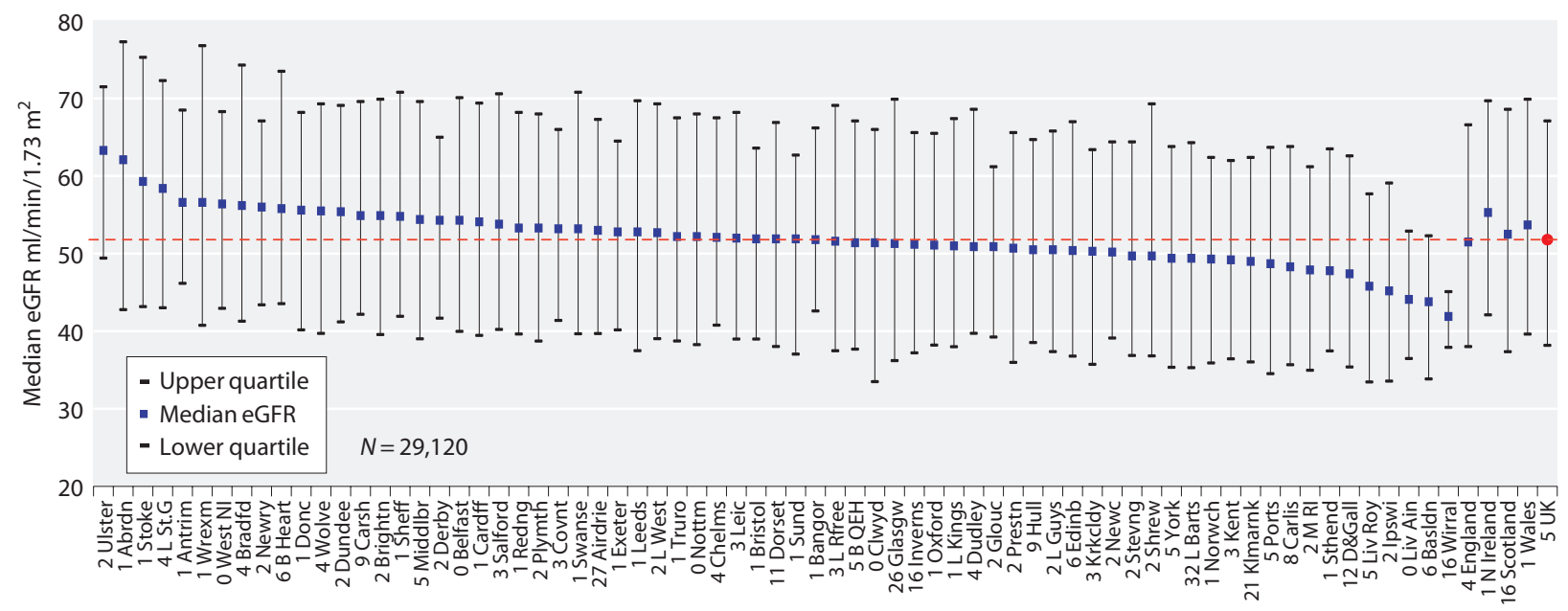

Centre

Fig. 3.2. Median eGFR in prevalent transplant patients by centre on $31 / 12 / 2015$

were excluded from analyses. For haemoglobin, estimated glomerular filtration rate (eGFR), corrected calcium, phosphate and blood pressure (BP), the latest value in quarter 3 or quarter 4 of 2015 was used.

\section{Estimated glomerular filtration rate (eGFR)}

For the purpose of eGFR calculation, the original 4-variable MDRD formula was used (with a constant of 186) to calculate eGFR from the serum creatinine concentration as reported by the centre (unless otherwise stated). A wide variety of creatinine assays are in use in clinical biochemistry laboratories in the $\mathrm{UK}$, and it is not possible to ensure that all measurements of creatinine concentration collected by the UKRR are harmonised. Patients with valid serum creatinine results but no ethnicity data were classed as White for the purpose of the eGFR calculation.

\section{One year post-transplant data}

Patients who received a renal transplant between 1st January 2008 and 31st December 2014 were assigned according to the renal centre in which they were transplanted. In a small number of instances, the first documented evidence of transplantation in a patient's record is from a timeline entry in data returned from a non-transplant centre, in these instances the patient was reassigned to the nearest transplant centre.

As this analysis is stratified by transplant type, and for some of the renal centres reporting of donor type to the UKRR is poor, donor-type used in this analysis was obtained from NHSBT.

Patients who had died or experienced graft failure within 12 months of transplantation were excluded from the analyses. Patients with more than one transplant during 2008-2014 were included as separate episodes provided each of the transplants functioned for a year.

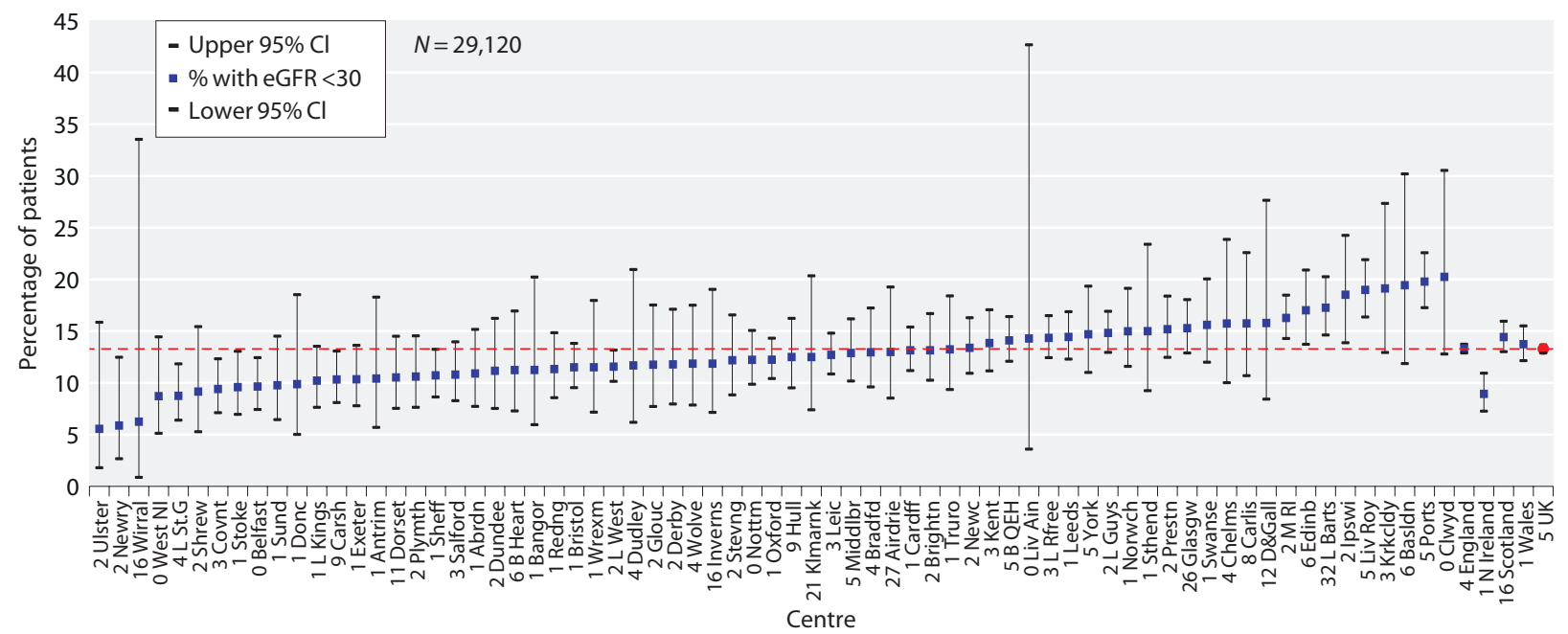

Fig. 3.3. Percentage of prevalent transplant patients by centre on $31 / 12 / 2015$ with eGFR $<30 \mathrm{ml} / \mathrm{min} / 1.73 \mathrm{~m}{ }^{2}$ 
For each patient, the most recent laboratory or blood pressure result for the relevant 4 th/5th quarter after renal transplantation was taken to be representative of the one year post-transplant outcome. Again, for the purpose of the eGFR calculation patients with valid serum creatinine results but missing ethnicity data were classed as White.

\section{Results and Discussion}

Post-transplant eGFR in prevalent transplant patients

When interpreting eGFR post-transplantation, it is important to remember that estimated GFR formulae only have a modest predictive performance in the transplant population [4]. Median eGFR in each centre and percentage of patients with eGFR $<30 \mathrm{ml} / \mathrm{min} / 1.73 \mathrm{~m}^{2}$ are shown in figures 3.2 and 3.3.

The median eGFR was $51.8 \mathrm{ml} / \mathrm{min} / 1.73 \mathrm{~m}^{2}$, with $13.3 \%$ of prevalent transplant recipients having an
eGFR $<30 \mathrm{ml} / \mathrm{min} / 1.73 \mathrm{~m}^{2}$, summarised by centre in table 3.10. Whilst local repatriation policies on timing of transfer of care for patients with failing transplants from transplant centres to referring centres might explain some of the differences, it is notable that both transplanting and non-transplanting centres feature at both ends of the scale in figure 3.3. The accuracy of the 4 -variable MDRD equation in estimating GFR $\geqslant 60 \mathrm{ml} / \mathrm{min} /$ $1.73 \mathrm{~m}^{2}$ is questionable [5], therefore a figure describing this is not included in this chapter.

Figure 3.4 shows the percentage of prevalent patients by centre with eGFR $<30 \mathrm{ml} / \mathrm{min} / 1.73 \mathrm{~m}^{2}$ as a funnel plot, enabling a more reliable comparison of outcomes between centres across the UK. The solid lines show the 2 standard deviation limits (95\%) and the dotted lines the limits for 3 standard deviations (99.9\%). With

Table 3.10. Percentage of prevalent transplant patients with eGFR $<30 \mathrm{ml} / \mathrm{min} / 1.73 \mathrm{~m}^{2}$ on $31 / 12 / 2015$

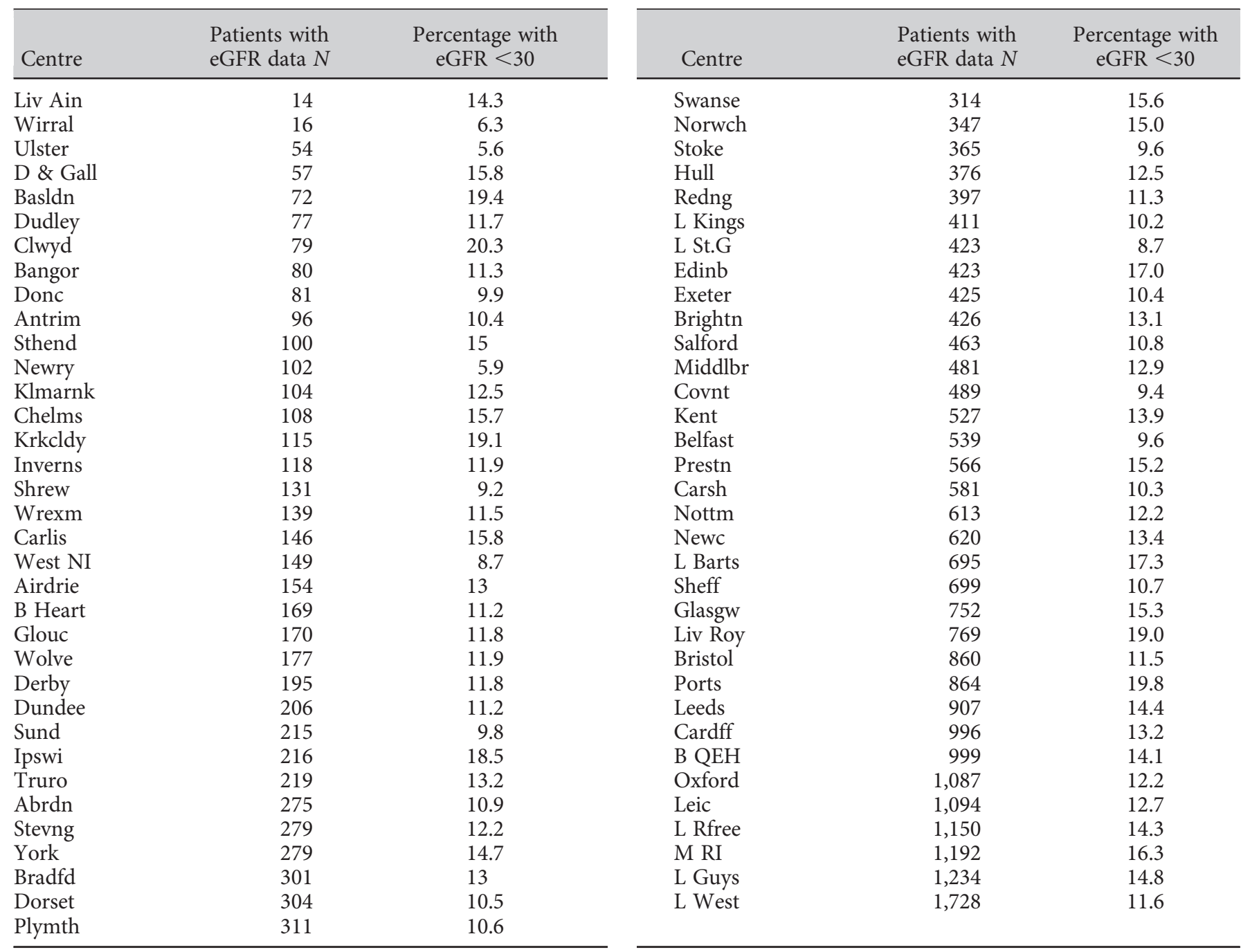




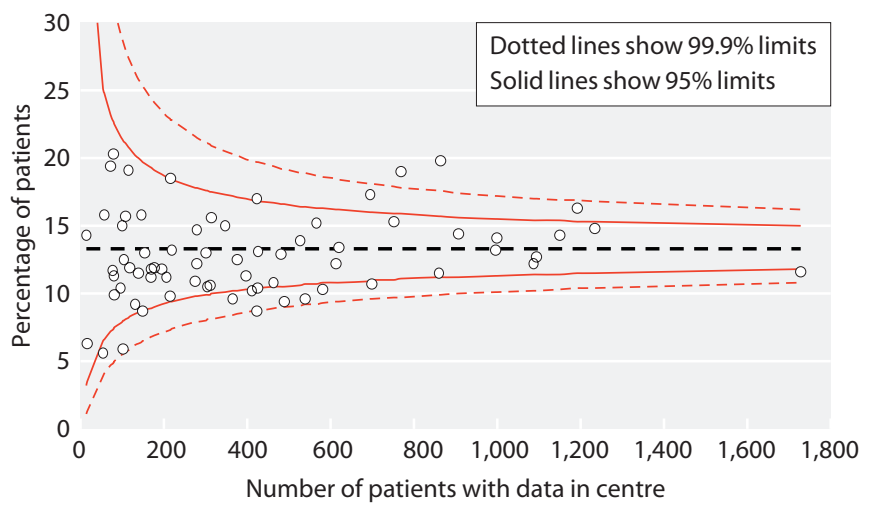

Fig. 3.4. Funnel plot of percentage of prevalent transplant patients with eGFR $<30 \mathrm{ml} / \mathrm{min} / 1.73 \mathrm{~m}^{2}$ by centre size on $31 / 12 / 2015$

69 centres included and a normal distribution, 3-4 centres would be expected to fall between the 95-99.9\% CI ( 1 in 20) and no centres should fall outside the 99.9\% limits.

There continued to be variation between centres; these data show over-dispersion with 15 centres falling outside the 95\% CI. Liverpool Royal and Portsmouth both fell outside the upper $99.9 \%$ CI suggesting a higher than expected proportion of patients with eGFR $<30 \mathrm{ml} /$ $\min / 1.73 \mathrm{~m}^{2}$.

eGFR in patients one year after transplantation

Graft function at one year post-transplantation may predict subsequent long-term graft outcome [6]. Figures $3.5 \mathrm{a}, 3.5 \mathrm{~b}$, and $3.5 \mathrm{c}$ show the median one-year post-transplant eGFR for patients transplanted between 2008-2014, by transplant type. Living kidney donation had the highest median eGFR at one year $(57.5 \mathrm{ml} / \mathrm{min} /$ $1.73 \mathrm{~m}^{2}$ ), followed by donation after brainstem death $\left(53.7 \mathrm{ml} / \mathrm{min} / 1.73 \mathrm{~m}^{2}\right)$ and donation after circulatory death $\left(50.4 \mathrm{ml} / \mathrm{min} / 1.73 \mathrm{~m}^{2}\right)$.

Figures 3.6a, 3.6b and 3.6c show one-year posttransplant eGFR by donor type and year of transplantation. There was no trend in eGFR over the time period for live kidney donation transplantation, donation after brainstem death or donation after circulatory death.

\section{Haemoglobin in prevalent transplant patients}

The Renal Association Anaemia guidelines recommend 'achieving a population distribution centred on a mean of $11 \mathrm{~g} / \mathrm{dl}$ with a range of 10-12 $\mathrm{g} / \mathrm{dl}$ ' [7] (equivalent to $110 \mathrm{~g} / \mathrm{L}$, range $100-120 \mathrm{~g} / \mathrm{L}$ ). However, many transplant patients with good transplant function will have haemoglobin concentrations $>120 \mathrm{~g} / \mathrm{L}$ without the use of erythropoiesis stimulating agents, and so it is inappropriate to audit performance using the higher limit.

A number of factors, including comorbidity, immunosuppressive medication, graft function, ACE inhibitor use, erythropoietin (EPO) use, intravenous or oral iron use, that affect centre-specific protocols for management of anaemia will affect haemoglobin concentrations in transplant patients. Most of these data are not collected by the UKRR and therefore caution must be used when interpreting analyses of haemoglobin attainment.

Figures $3.7 \mathrm{a}$ and $3.7 \mathrm{~b}$ report centre results stratified according to graft function as estimated by eGFR. The percentage of prevalent transplant patients achieving $\mathrm{Hb} \geqslant 100 \mathrm{~g} / \mathrm{L}$ in each centre, stratified by eGFR, is displayed in figures $3.8 \mathrm{a}$ and $3.8 \mathrm{~b}$.

Figure 3.9 describes the percentage of prevalent patients by centre with haemoglobin $<100 \mathrm{~g} / \mathrm{L}$ as a funnel plot enabling more reliable comparison of outcomes between centres across the UK. With 69 centres included and a normal distribution, 3-4 centres would be expected to fall between the $95 \%-99.9 \%$ CI ( 1 in 20 ) and no centres should fall outside the $99.9 \%$ CI purely as a chance event.

One centre (London St Bartholomew's) fell outside the upper 99.9\% CI and two further centres (London Guys and London Kings) fell outside the upper 95\% CI indicating a higher than predicted proportion of transplant patients not achieving the haemoglobin target. Seven centres fell outside the lower $99.9 \% \mathrm{CI}$, indicating they performed better than expected with fewer than predicted patients having a haemoglobin $<100 \mathrm{~g} / \mathrm{L}$.

\section{Blood pressure in prevalent transplant patients}

The UK Renal Association (RA) guideline for the care of kidney transplant recipients recommends that 'Blood pressure should be $<130 / 80 \mathrm{mmHg}$ (or $<125$ / $75 \mathrm{mmHg}$ if proteinuria)' [8]. This blood pressure (BP) target is the same as that used in previous annual reports. Completeness for blood pressure data returns was variable with some centres unable to report. Data from 34 centres with $>50 \%$ data returns were included in the analysis. Despite this restriction, caution needs to be exercised in interpretation of these results because of the volume of missing data and potential bias, (e.g. a centre may be more likely to record and report blood pressure data electronically in patients with poor $\mathrm{BP}$ control). 


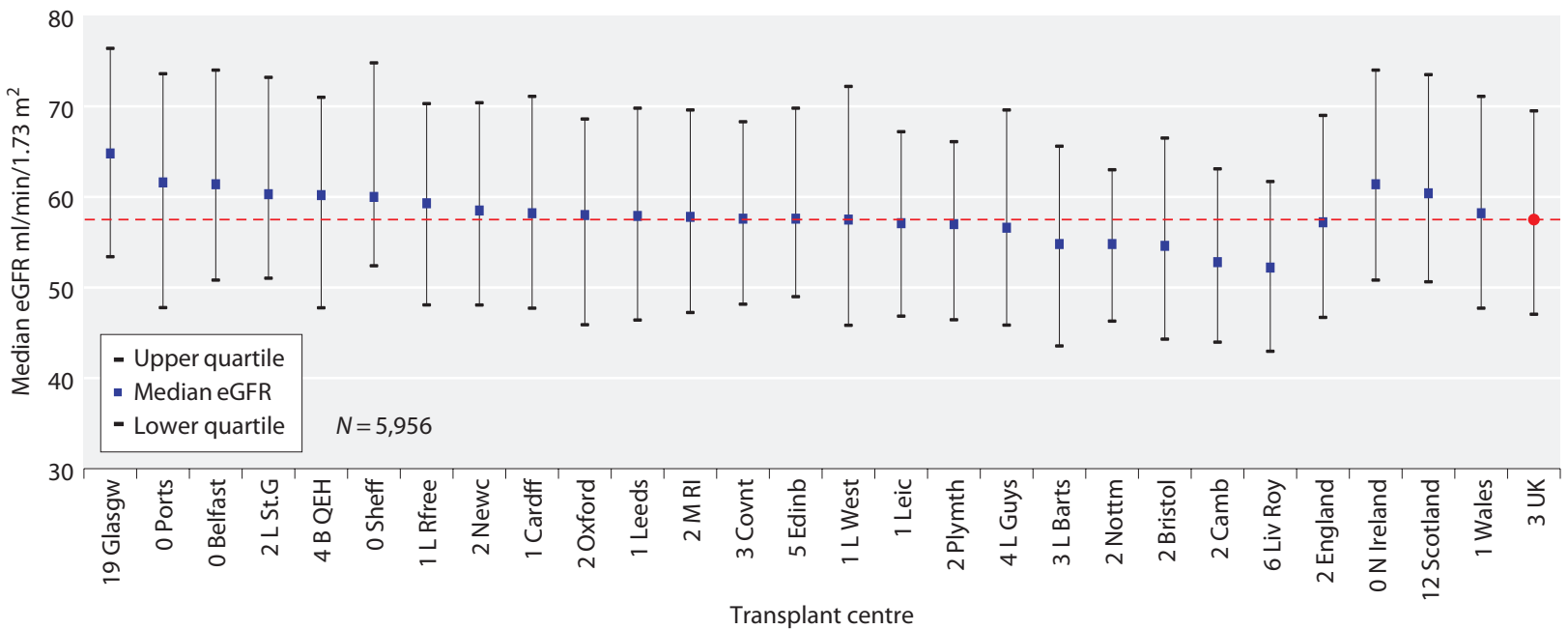

Fig. 3.5a. Median eGFR one year post-live donor transplant by transplant centre 2008-2014

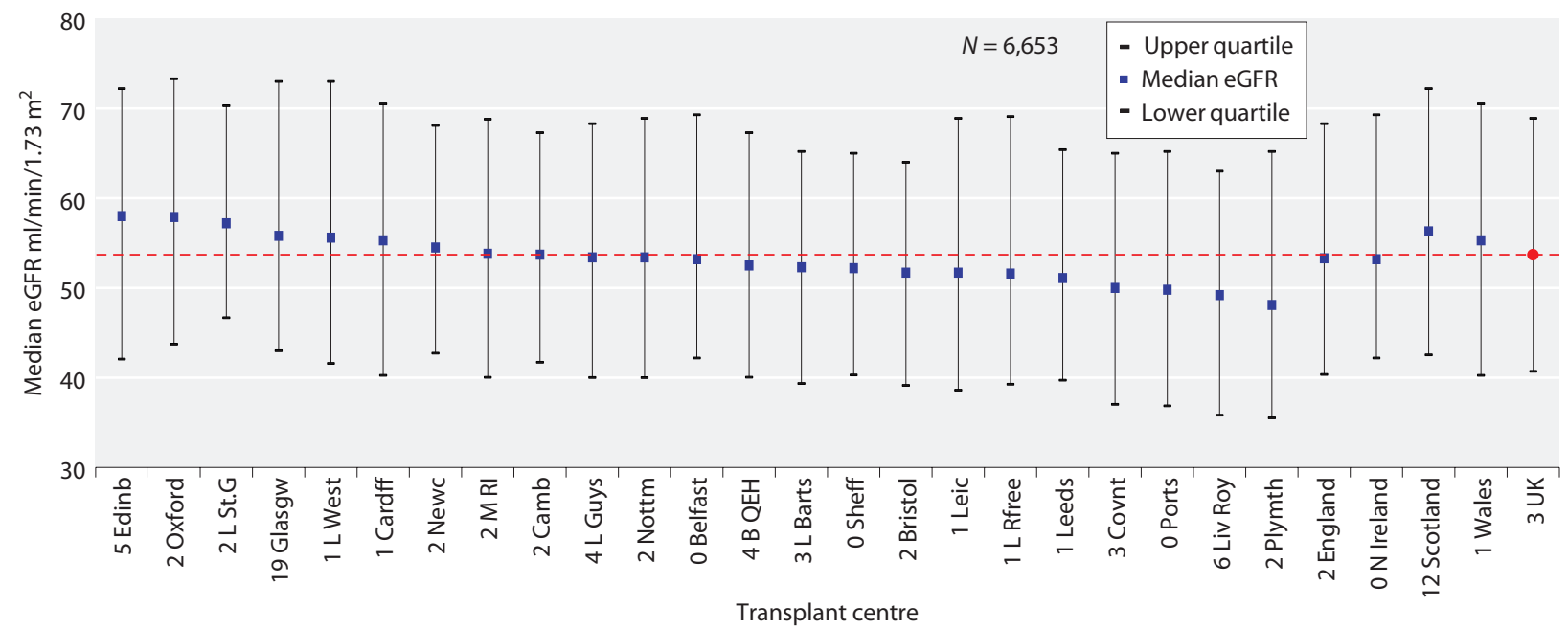

Fig. 3.5b. Median eGFR one year post-brainstem death donor transplant by transplant centre 2008-2014

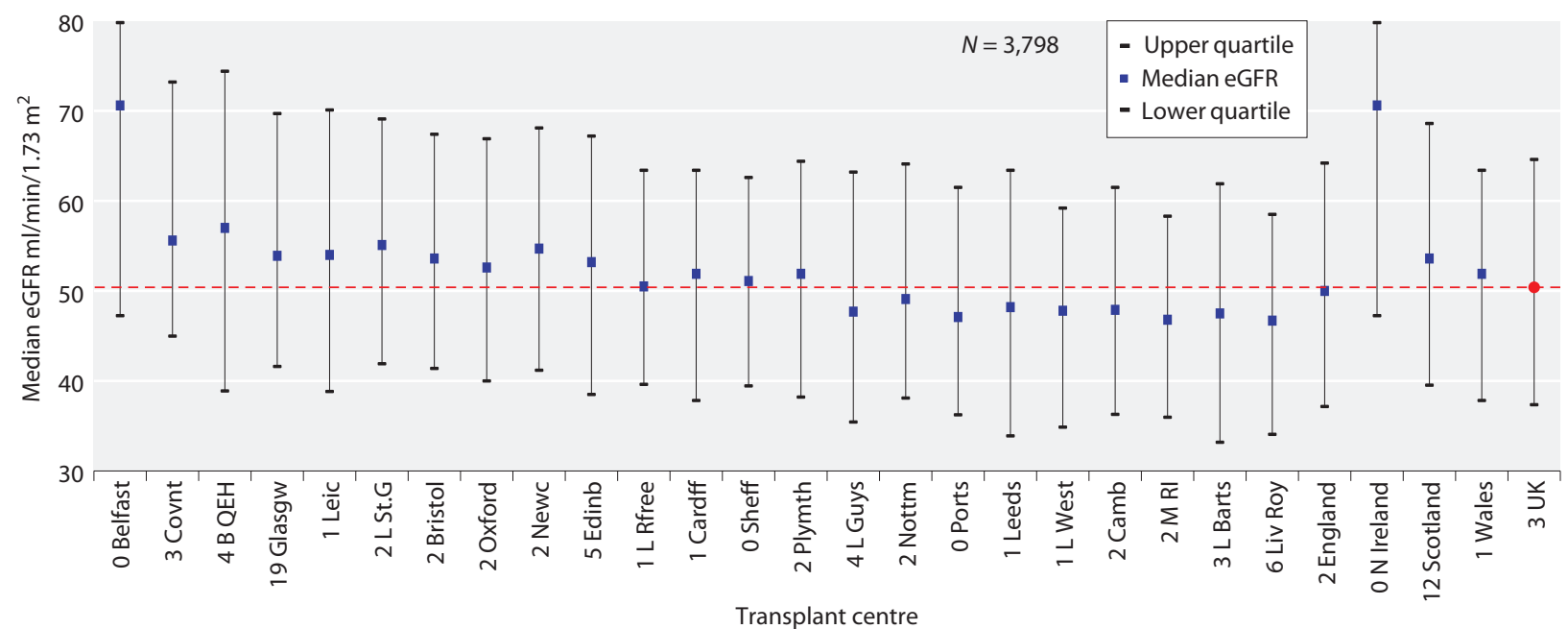

Fig. 3.5c. Median eGFR one year post-circulatory death donor transplant by transplant centre 2008-2014 


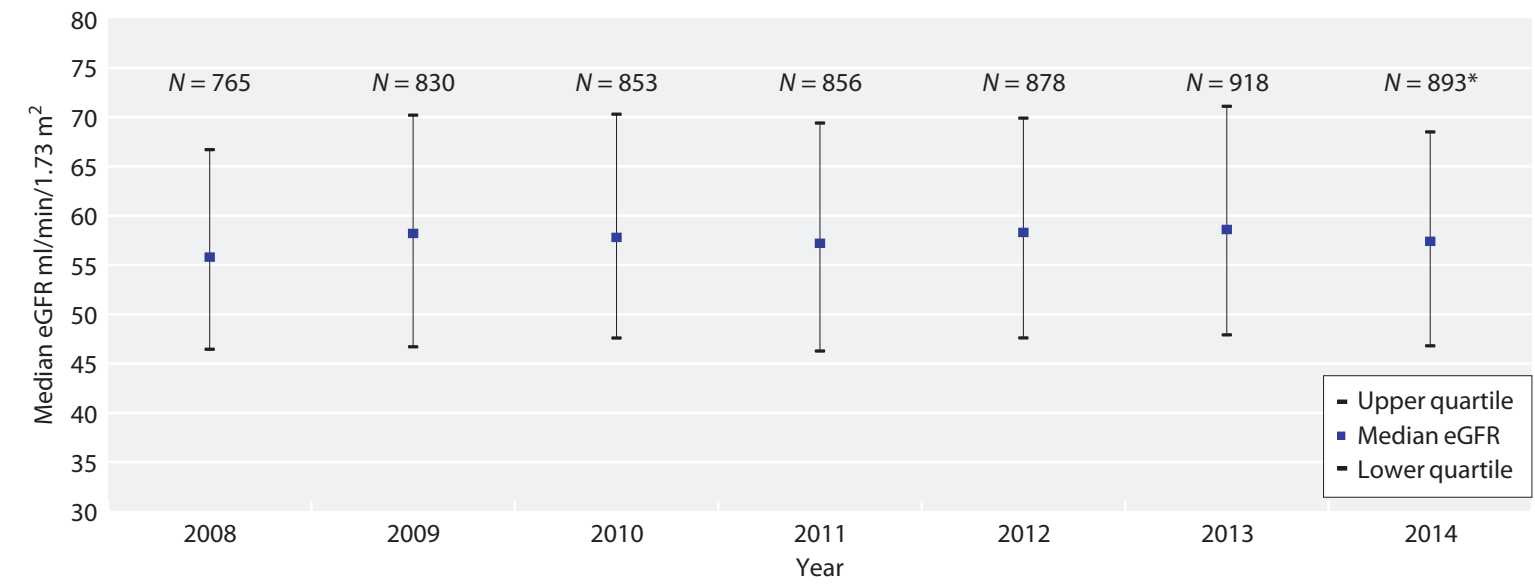

Fig. 3.6a. Median eGFR one year post-live donor transplant by year of transplantation 2008-2014

*This number does not include live-donor transplants performed in 2014 that were followed-up in Cambridge in 2015, as Cambridge was unable to submit data for 2015

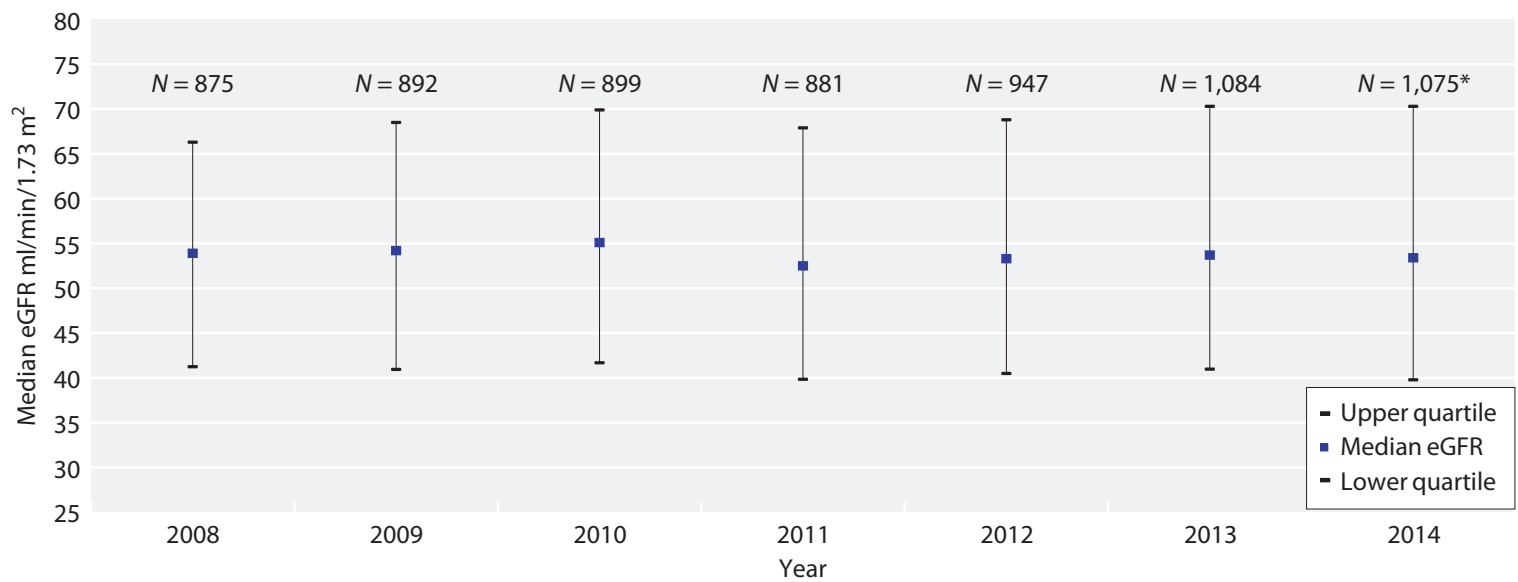

Fig. 3.6b. Median eGFR one year post-brainstem death donor transplant by year of transplantation 2008-2014

*This number does not include post-brainstem death donor transplants performed in 2014 that were followed-up in Cambridge in 2015, as Cambridge was unable to submit data for 2015

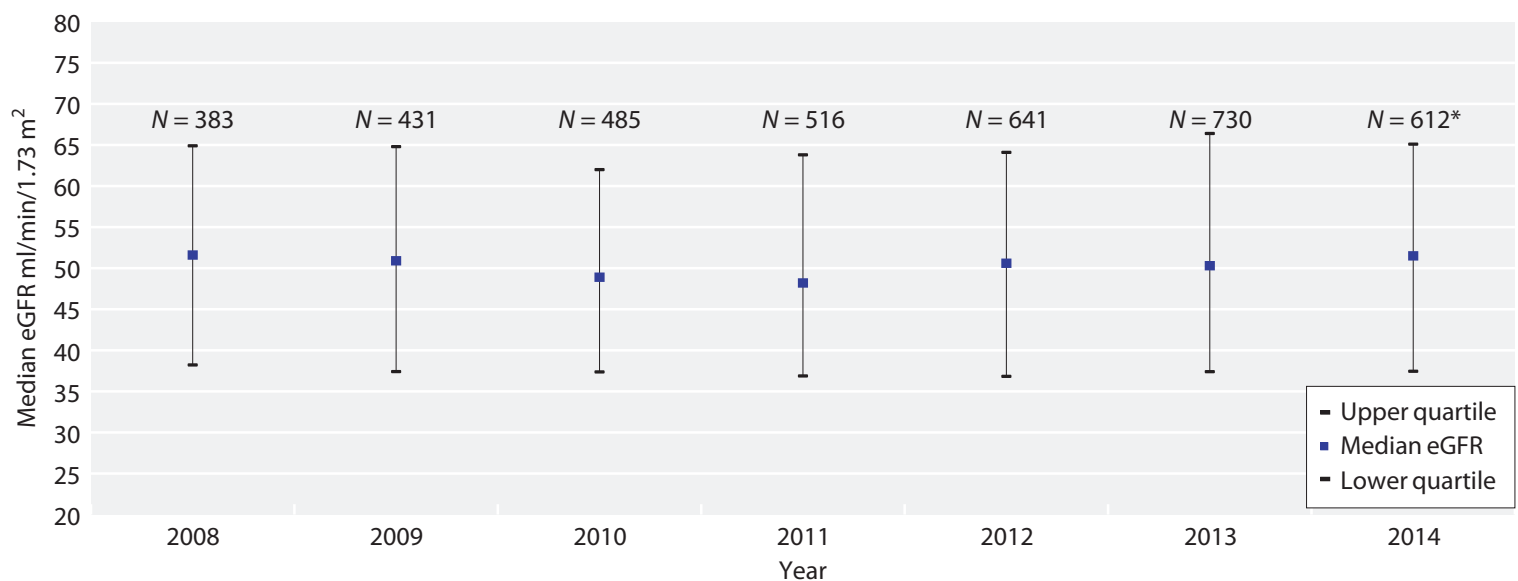

Fig. 3.6c. Median eGFR one year post-circulatory death donor transplant by year of transplantation 2008-2014

*This number does not include post-circulatory death donor transplants performed in 2014 that were followed-up in Cambridge in 2015, as Cambridge was unable to submit data for 2015 


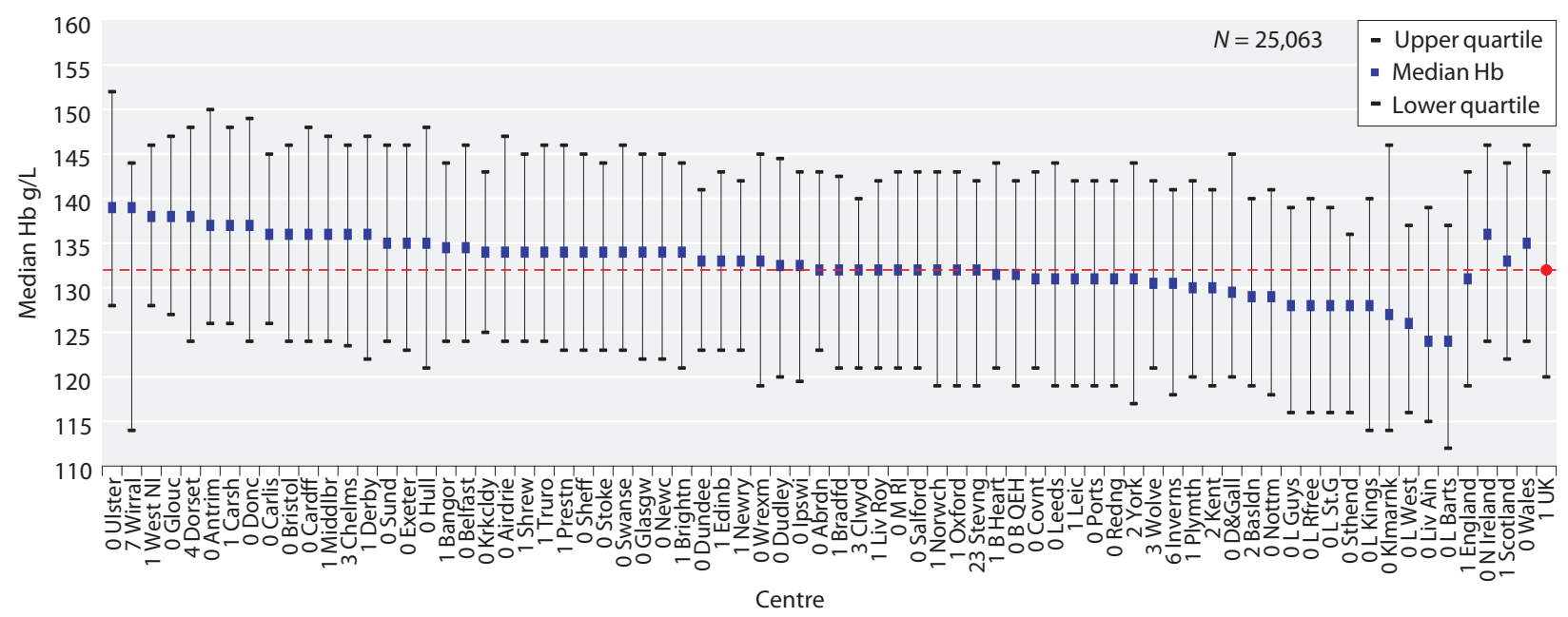

Fig. 3.7a. Median haemoglobin for prevalent transplant patients with eGFR $\geqslant 30 \mathrm{ml} / \mathrm{min} / 1.73 \mathrm{~m}^{2}$ by centre on $31 / 12 / 2015$

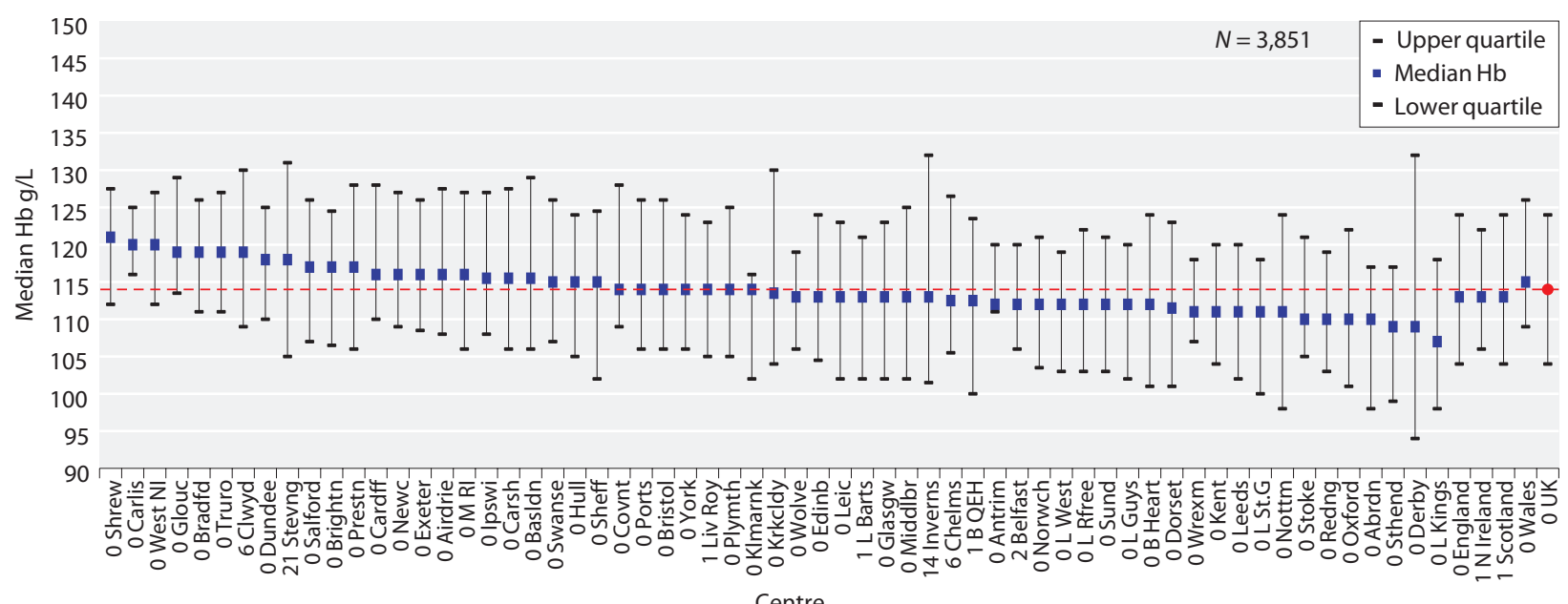

Fig. 3.7b. Median haemoglobin for prevalent transplant patients with eGFR $<30 \mathrm{ml} / \mathrm{min} / 1.73 \mathrm{~m}^{2}$ by centre on $31 / 12 / 2015$

Figures $3.10 \mathrm{a}$ and $3.10 \mathrm{~b}$ show the percentage of patients with a blood pressure of $<130 / 80 \mathrm{mmHg}$, by eGFR. The percentage of patients with BP $<130 / 80$ (systolic BP $<130$ and diastolic $\mathrm{BP}<80 \mathrm{mmHg}$ ) was higher $(26.6 \%$ vs. $21.8 \%)$ in those with better renal function (eGFR $\geqslant 30 \mathrm{ml} / \mathrm{min} / 1.73 \mathrm{~m}^{2}$ ).

\section{Analysis of prevalent patients by CKD stage}

\section{Introduction}

Approximately $2.7 \%$ of prevalent transplant patients returned to dialysis in 2015 , a similar percentage to that seen over the last few years. Amongst patients with native chronic kidney disease, late presentation is associated with poor outcomes, largely attributable to lack of specialist management of anaemia, acidosis, hyperphosphataemia and to inadequate advance preparation for dialysis. Transplant recipients on the other hand, are almost always followed up regularly in specialist transplant or renal clinics and it would be reasonable to expect patients with failing grafts to receive appropriate care and therefore have many of their modifiable risk factors addressed before complete graft failure and return to dialysis.

\section{Methods}

The transplant cohort consisted of prevalent transplant recipients as on 31st December 2015 and patients were classified according to the KDIGO staging criteria with the suffix of ' $\mathrm{T}$ ' to represent their transplant status. Patients with missing ethnicity information were classified as White for the purpose of calculating eGFR. Prevalent dialysis patients, except those who commenced dialysis 


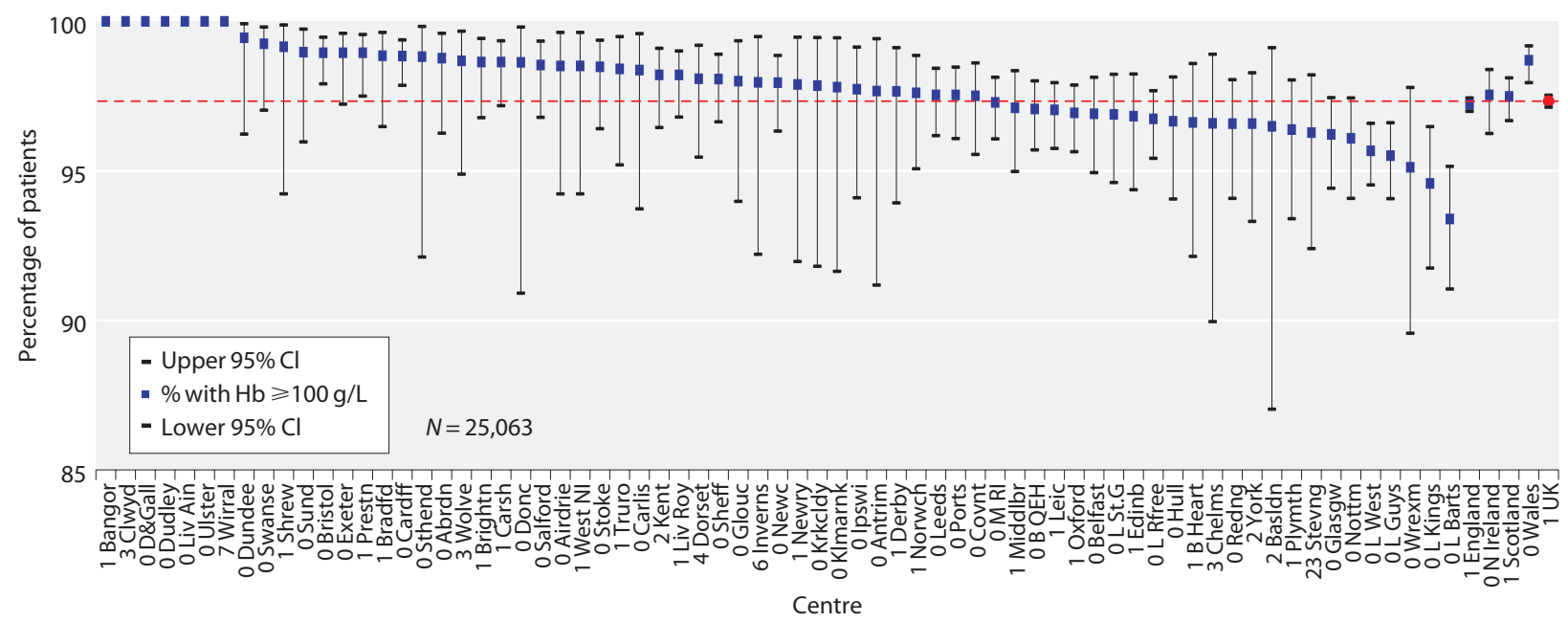

Fig. 3.8a. Percentage of prevalent transplant patients with eGFR $\geqslant 30 \mathrm{ml} / \mathrm{min} / 1.73 \mathrm{~m}^{2}$ achieving haemoglobin $\geqslant 100 \mathrm{~g} / \mathrm{L}$ by centre on $31 / 12 / 2015$

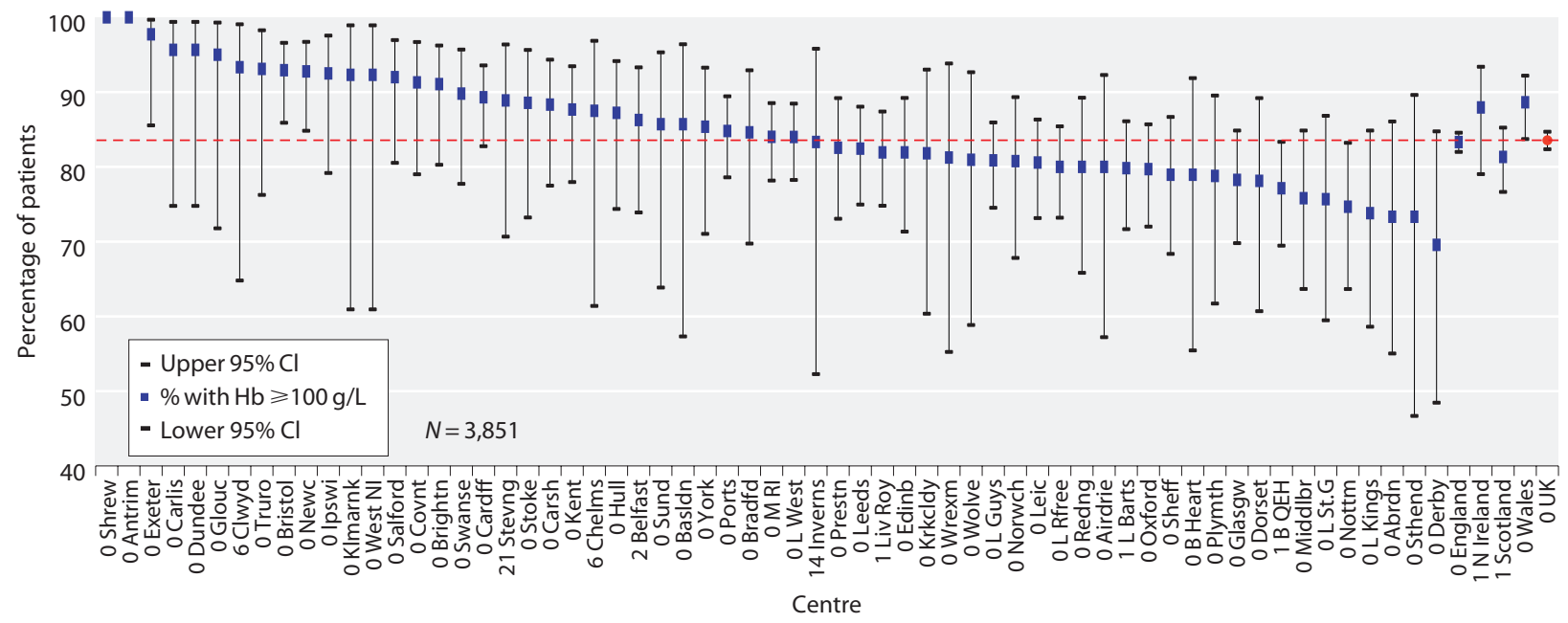

Fig. 3.8b. Percentage of prevalent transplant patients with eGFR $<30 \mathrm{ml} / \mathrm{min} / 1.73 \mathrm{~m}^{2}$ achieving haemoglobin $\geqslant 100 \mathrm{~g} / \mathrm{L}$ by centre on $31 / 12 / 2015$

in 2015 , comprised the comparison dialysis cohort $(N=21,367)$ including 2,163 peritoneal dialysis patients. Only patients on peritoneal dialysis were considered when examining differences in serum phosphate between transplant recipients and dialysis patients. For both the transplant and dialysis cohorts, the analysis used the most recent available value from the last two quarters of the 2015 laboratory data. Scottish centres were excluded from blood pressure, cholesterol and PTH analyses as corresponding data were not provided.

\section{Results and Discussion}

Table 3.11 shows that $13.3 \%$ of the prevalent transplant population ( 3,868 patients), had moderate to advanced renal impairment of eGFR $<30 \mathrm{ml} / \mathrm{min} /$ $1.73 \mathrm{~m}^{2}$. The table also demonstrates that patients with failing grafts had poorer blood pressure control, and

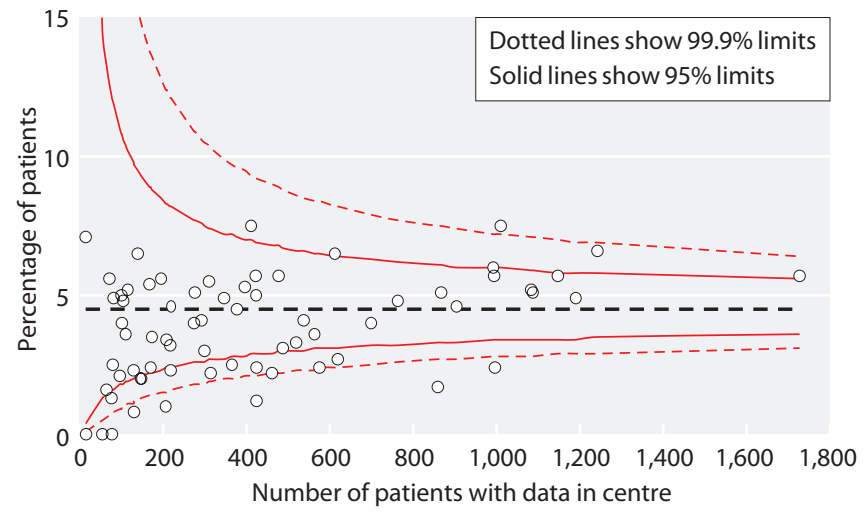

Fig. 3.9. Funnel plot of percentage of prevalent transplant patients with haemoglobin $<100 \mathrm{~g} / \mathrm{L}$ by centre size on $31 / 12 / 2015$ 


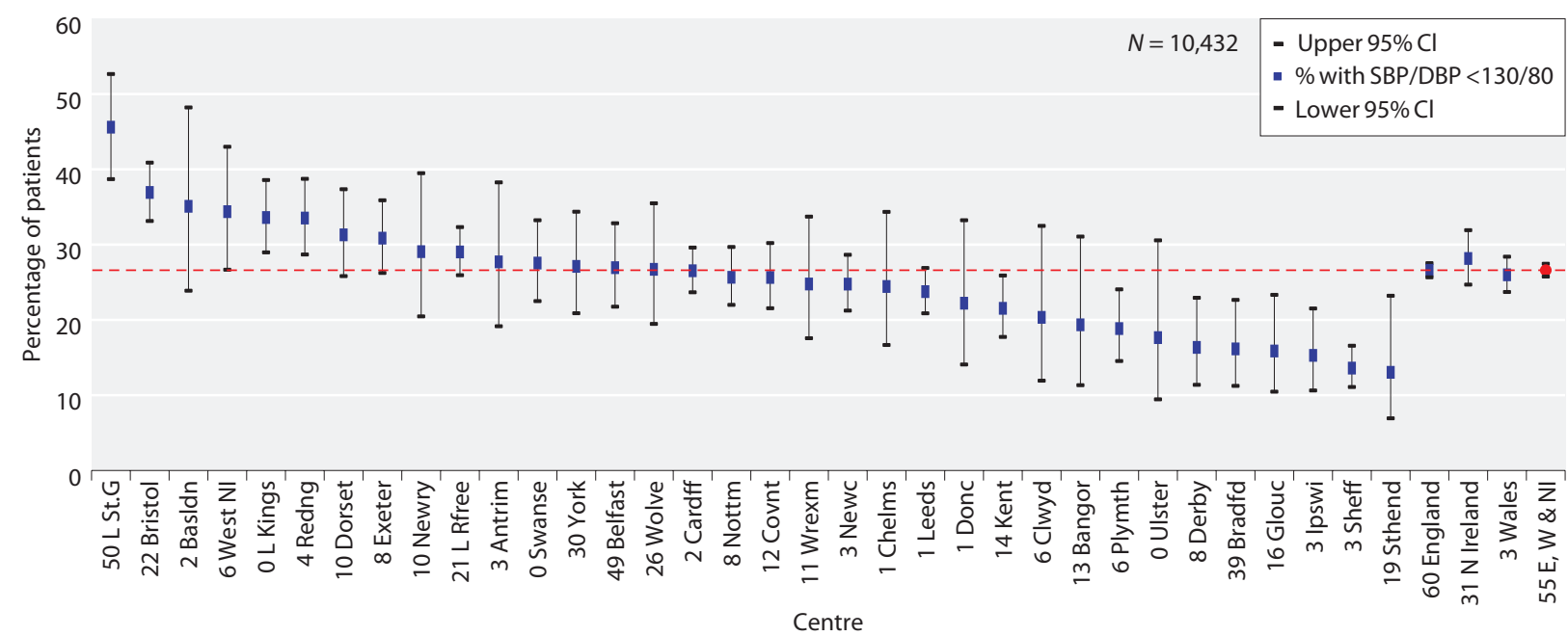

Fig. 3.10a. Percentage of prevalent transplant patients with eGFR $\geqslant 30 \mathrm{ml} / \mathrm{min} / 1.73 \mathrm{~m}^{2}$ achieving blood pressure of $<130 / 80 \mathrm{mmHg}$ by centre on $31 / 12 / 2015$

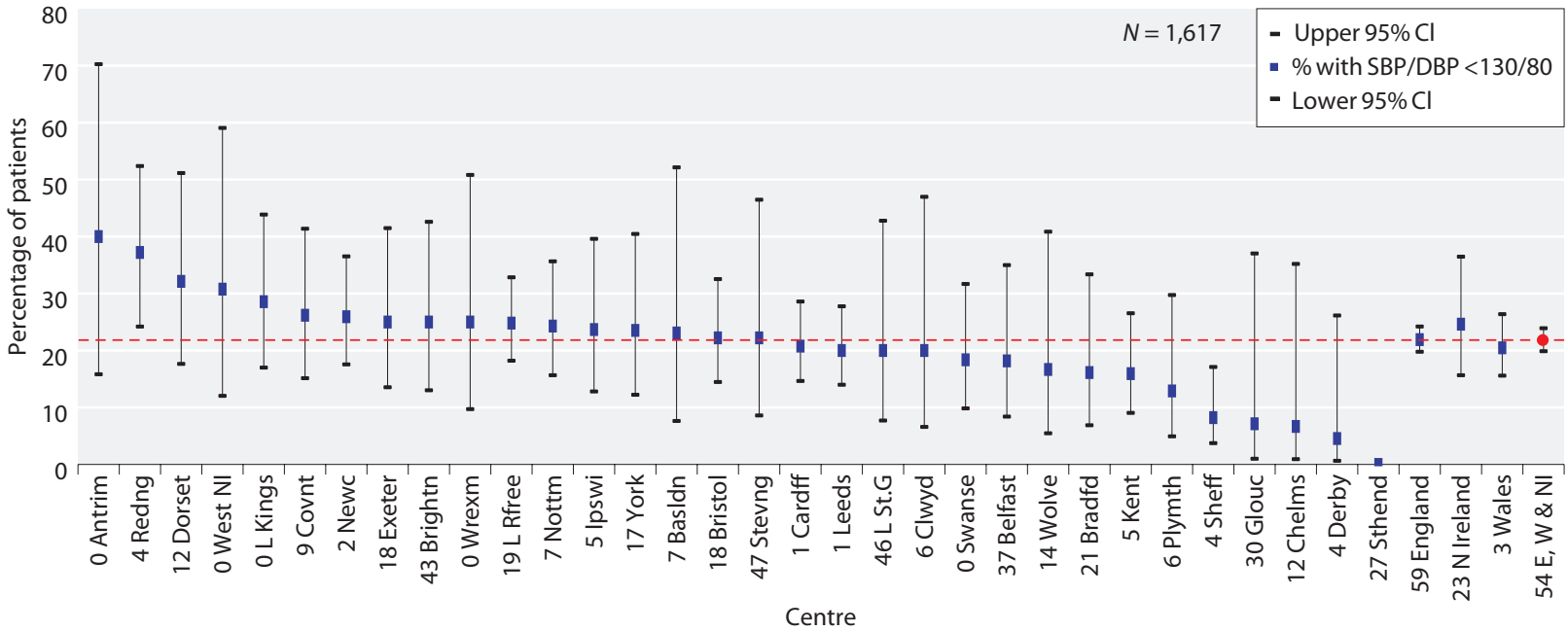

Fig. 3.10b. Percentage of prevalent transplant patients with eGFR $<30 \mathrm{ml} / \mathrm{min} / 1.73 \mathrm{~m}^{2}$ achieving blood pressure of $<130 / 80 \mathrm{mmHg}$ by centre on $31 / 12 / 2015$

achieved UK Renal Association standards for some key biochemical and clinical outcome variables less often than dialysis patients. This substantial group of patients continues to represent a challenge, and improved predialysis management should allow for timely re-listing for transplantation if appropriate, and a smooth transition to another renal replacement modality.

\section{eGFR slope analysis}

\section{Introduction}

The gradient of deterioration in eGFR (slope) may predict patients likely to have early graft failure. The eGFR slope and its relationship to specific patient characteristics are presented here. 
Table 3.11. Analysis by CKD stage for prevalent transplant patients compared with prevalent dialysis patients on $31 / 12 / 2015$

\begin{tabular}{|c|c|c|c|c|c|}
\hline & $\begin{array}{l}\text { Stage } 1-2 \mathrm{~T} \\
\quad(\geqslant 60)\end{array}$ & $\begin{array}{l}\text { Stage } 3 \mathrm{~T} \\
(30-59)\end{array}$ & $\begin{array}{l}\text { Stage } 4 \mathrm{~T} \\
(15-29)\end{array}$ & $\begin{array}{l}\text { Stage } 5 \mathrm{~T} \\
(<15)\end{array}$ & Stage 5D \\
\hline $\begin{array}{l}\text { Number of patients } \\
\% \text { of patients }\end{array}$ & $\begin{array}{l}10,379 \\
35.6\end{array}$ & $\begin{array}{l}14,886 \\
51.1\end{array}$ & $\begin{array}{l}3,394 \\
11.7\end{array}$ & $\begin{array}{l}474 \\
1.6\end{array}$ & 21,367 \\
\hline $\begin{array}{l}\text { eGFR } \mathbf{m}, / \mathrm{min} / 1.73 \mathrm{~m}^{2 \mathrm{a}} \\
\text { mean } \pm \mathrm{SD} \\
\text { Median }\end{array}$ & $\begin{array}{c}77.1 \pm 15.5 \\
73.2\end{array}$ & $\begin{array}{c}45.7 \pm 8.3 \\
46.1\end{array}$ & $\frac{23.8 \pm}{24.3}$ & $\begin{array}{c}12.0 \pm 2.1 \\
12.4\end{array}$ & \\
\hline $\begin{array}{l}\text { Systolic BP mmHg } \\
\text { mean } \pm \text { SD } \\
\% \geqslant 130\end{array}$ & $\begin{array}{c}133.8 \pm 16.2 \\
60.1\end{array}$ & $\begin{array}{c}135.8 \pm 17.0 \\
64.3\end{array}$ & $\begin{array}{c}139.6 \pm 19.2 \\
70.1\end{array}$ & $\begin{array}{c}144.6 \pm 21.0 \\
76.8\end{array}$ & $\begin{array}{c}132.7 \pm 24.8 \\
52.3\end{array}$ \\
\hline $\begin{array}{l}\text { Diastolic BP } \mathbf{m m H g} \\
\text { mean } \pm \text { SD } \\
\% \geqslant 80\end{array}$ & $79.1 \pm 10.4$ & $\begin{array}{c}78.5 \pm 10.7 \\
48.0\end{array}$ & $\begin{array}{c}79.1 \pm 11.9 \\
49.7\end{array}$ & $80.3 \pm 12.7$ & $68.7 \pm 14.9$ \\
\hline $\begin{array}{l}\text { Cholesterol } \mathbf{~ m m o l} / \mathrm{L} \\
\text { mean } \pm \mathrm{SD} \\
\% \geqslant 4\end{array}$ & $\begin{array}{l}4.5 \pm 1.0 \\
68.0\end{array}$ & $\begin{array}{l}4.6 \pm 1.1 \\
71.7\end{array}$ & $\begin{array}{l}4.7 \pm 1.2 \\
71.1\end{array}$ & $\begin{array}{l}4.7 \pm 1.3 \\
68.8\end{array}$ & $\begin{array}{l}3.9 \pm 1.1 \\
42.4\end{array}$ \\
\hline $\begin{array}{l}\text { Haemoglobin } \mathbf{g} / \mathbf{L} \\
\text { mean } \pm \mathrm{SD} \\
\%<100.0\end{array}$ & $136.7 \pm 15.9$ & $128.0 \pm \frac{ \pm}{3.6} 16.5$ & $\begin{array}{c}115.3 \pm 15.4 \\
14.3\end{array}$ & $\begin{array}{c}105.2 \pm 13.9 \\
32.1\end{array}$ & $\begin{array}{c}110.3 \pm 13.6 \\
19.5\end{array}$ \\
\hline $\begin{array}{l}\text { Phosphate } \mathbf{~ m m o l} / \mathbf{L}^{\mathbf{b}} \\
\text { mean } \pm \mathrm{SD} \\
\%>1.7\end{array}$ & $\begin{array}{c}0.9 \pm 0.2 \\
0.1\end{array}$ & $1.0 \pm 0.2$ & $1.1 \underset{2.4}{ \pm} 0.3$ & $\begin{array}{l}1.5 \pm 0.4 \\
25.8\end{array}$ & $\begin{array}{l}1.6 \pm 0.4 \\
36.0\end{array}$ \\
\hline $\begin{array}{l}\text { Corrected calcium mmol/L } \\
\text { mean } \pm \text { SD } \\
\%>2.5 \\
\%<2.2\end{array}$ & $\begin{array}{l}2.4 \pm 0.1 \\
26.7 \\
3.3\end{array}$ & $\begin{array}{l}2.4 \pm 0.1 \\
26.7 \\
3.8\end{array}$ & $\begin{array}{l}2.4 \pm 0.2 \\
19.4 \\
8.1\end{array}$ & $\begin{array}{l}2.4 \pm 0.2 \\
18.7 \\
15.1\end{array}$ & $\begin{array}{l}2.4 \pm 0.2 \\
15.9 \\
16.8\end{array}$ \\
\hline $\begin{array}{l}\text { PTH pmol/L } \\
\text { Median } \\
\%>72\end{array}$ & $\begin{array}{l}8.7 \\
0.3\end{array}$ & $\begin{array}{r}10.1 \\
0.7\end{array}$ & $\begin{array}{r}16.8 \\
3.8\end{array}$ & $\begin{array}{l}32.6 \\
20.6\end{array}$ & $\begin{array}{l}33.5 \\
19.6\end{array}$ \\
\hline
\end{tabular}

${ }^{\text {a }}$ Prevalent transplant patients with no ethnicity data were classed as White

${ }^{\mathrm{b}}$ Only PD patients included in stage $5 \mathrm{D}, N=2,163$

\section{Methods}

All UK patients aged $\geqslant 18$ years receiving their first renal transplant between 1st January 2004 and 31st December 2013, were considered for inclusion. A minimum duration of 18 months graft function was required and three or more creatinine measurements from the second year of graft function onwards were used to plot eGFR slope. If a transplant failed but there were at least three creatinine measurements between one year post-transplant and graft failure, the patient was included but no creatinine measurements after the quarter preceding the recorded date of transplant failure were analysed.

Slopes were calculated using linear regression, assuming linearity, and the effect of age, ethnicity, gender, diabetes, donor type, year of transplant and current transplant status were analysed. $P$ values were calculated using the Kruskal-Wallis test. eGFR was calculated using the CKD-EPI equation and results expressed as $\mathrm{ml} / \mathrm{min} / 1.73 \mathrm{~m}^{2} /$ year. The CKD-EPI equation was used in preference to the MDRD formula as it is thought to have a greater degree of accuracy at higher levels of eGFR [9].

Outcomes in UK renal transplant recipients in 2015

\section{Results and Discussion}

The study cohort consisted of 17,357 patients. The median GFR slope was $-0.56 \mathrm{ml} / \mathrm{min} / 1.73 \mathrm{~m}^{2} /$ year (table 3.12). The gradient was steeper for Black recipients $\left(-1.01 \mathrm{ml} / \mathrm{min} / 1.73 \mathrm{~m}^{2} /\right.$ year$)$, in keeping with previously published data suggesting poorer outcomes for this group [10].

There was no statistically significant difference in eGFR slope in recipients of deceased donor kidneys $\left(-0.57 \mathrm{ml} / \mathrm{min} / 1.73 \mathrm{~m}^{2} /\right.$ year$)$ compared to patients who received organs from live donors $(-0.54 \mathrm{ml} / \mathrm{min} /$ $1.73 \mathrm{~m}^{2} /$ year), although there was a significant difference in the eGFR slope in recipients of deceased cardiac death kidneys $\left(-0.33 \mathrm{ml} / \mathrm{min} / 1.73 \mathrm{~m}^{2} /\right.$ year, $\left.P<0.001\right)$. Female patients had a steeper slope $(-0.98 \mathrm{ml} / \mathrm{min} /$ $1.73 \mathrm{~m}^{2} /$ year $)$ than males $\left(-0.33 \mathrm{ml} / \mathrm{min} / 1.73 \mathrm{~m}^{2} /\right.$ year $)$,

Nephron 2017;137(suppl1):73-102 
Table 3.12. Differences in median eGFR slope between subgroups of prevalent transplant patients

\begin{tabular}{|c|c|c|c|c|c|c|}
\hline Patients characteristics & & $N$ & Median slope & Lower quartile & Upper quartile & $\mathrm{p}$-value \\
\hline Age at transplant & $\begin{array}{c}<40 \\
40-55 \\
>55\end{array}$ & $\begin{array}{l}4,936 \\
6,618 \\
5,803 \\
\end{array}$ & $\begin{array}{l}-1.07 \\
-0.38 \\
-0.39 \\
\end{array}$ & $\begin{array}{l}-4.14 \\
-2.70 \\
-2.68 \\
\end{array}$ & $\begin{array}{l}0.95 \\
1.46 \\
1.59 \\
\end{array}$ & $<0.0001$ \\
\hline Ethnicity & $\begin{array}{l}\text { Asian } \\
\text { Black } \\
\text { Other } \\
\text { White }\end{array}$ & $\begin{array}{r}1,729 \\
1,083 \\
489 \\
13,205\end{array}$ & $\begin{array}{l}-0.94 \\
-1.01 \\
-0.81 \\
-0.47\end{array}$ & $\begin{array}{l}-4.29 \\
-4.16 \\
-3.79 \\
-2.82\end{array}$ & $\begin{array}{l}1.30 \\
1.41 \\
1.36 \\
1.36\end{array}$ & $<0.0001$ \\
\hline Gender & $\begin{array}{c}\text { Male } \\
\text { Female }\end{array}$ & $\begin{array}{r}10,678 \\
6,679 \\
\end{array}$ & $\begin{array}{l}-0.33 \\
-0.98 \\
\end{array}$ & $\begin{array}{l}-2.69 \\
-3.63 \\
\end{array}$ & $\begin{array}{l}1.53 \\
1.09 \\
\end{array}$ & $<0.0001$ \\
\hline Diabetes & $\begin{array}{l}\text { No-diabetes } \\
\text { Diabetes }\end{array}$ & $\begin{array}{r}14,679 \\
2,541 \\
\end{array}$ & $\begin{array}{l}-0.46 \\
-1.15 \\
\end{array}$ & $\begin{array}{l}-2.88 \\
-4.12 \\
\end{array}$ & $\begin{array}{l}1.41 \\
0.98 \\
\end{array}$ & $<0.0001$ \\
\hline Donor & $\begin{array}{l}\text { Deceased } \\
\text { Live }\end{array}$ & $\begin{array}{r}11,211 \\
6,146 \\
\end{array}$ & $\begin{array}{l}-0.57 \\
-0.54 \\
\end{array}$ & $\begin{array}{l}-3.14 \\
-2.95 \\
\end{array}$ & $\begin{array}{l}1.37 \\
1.36 \\
\end{array}$ & 0.6 \\
\hline $\begin{array}{l}\text { Status of transplant } \\
\text { at end of follow-up }\end{array}$ & $\begin{array}{c}\text { Died } \\
\text { Failed } \\
\text { Re-transplanted } \\
\text { Functioning }\end{array}$ & $\begin{array}{r}1,261 \\
1,306 \\
70 \\
14,720\end{array}$ & $\begin{array}{l}-0.75 \\
-6.32 \\
-3.93 \\
-0.28\end{array}$ & $\begin{array}{r}-3.95 \\
-12.06 \\
-7.31 \\
-2.36\end{array}$ & $\begin{array}{r}1.83 \\
-3.03 \\
-1.85 \\
1.53\end{array}$ & $<0.0001$ \\
\hline
\end{tabular}

as did patients with diabetes $\left(-1.15 \mathrm{ml} / \mathrm{min} / 1.73 \mathrm{~m}^{2} /\right.$ year) compared to patients without $(-0.46 \mathrm{ml} / \mathrm{min} /$ $1.73 \mathrm{~m}^{2} /$ year). The slope was steeper in younger recipients, possibly reflecting differences in causes of graft failure. As might be expected, the steepest slope was in patients where the transplant subsequently failed. This analysis has assumed linearity of progression of fall in GFR and further work is ongoing to characterise the patterns of progression more precisely.

\section{Cause of death in transplant recipients}

\section{Introduction}

Differences in causes of death between dialysis and transplant patients may be expected due to selection for transplantation and use of immunosuppression. Chapter 5 includes a more detailed discussion on cause of death in dialysis patients.

\section{Methods}

The cause of death is sent by renal centres as an ERA-EDTA registry code. These have been grouped into the following categories: cardiac disease, cerebrovascular disease, infection, malignancy, treatment withdrawal, other and uncertain.

Some centres have high data returns to the UKRR regarding cause of death, whilst others return no information. Provision of this information is not mandatory. Analysis of prevalent patients included all those aged over 18 years and receiving RRT on 1st January 2015.

\section{Results and Discussion}

Table 3.13 and figure 3.11 show the differences in the cause of death between prevalent dialysis and transplant patients. Table 3.14 shows the cause of death for prevalent transplant patients by age.

Death due to cardiovascular disease was less common in transplanted patients than in dialysis patients, perhaps reflecting the lower age of the transplanted patients, and cardiovascular screening undertaken during transplant work-up; transplant recipients are a pre-selected lower 
Table 3.13. Cause of death by modality in prevalent RRT patients on $1 / 1 / 2015$, who died in 2015

\begin{tabular}{|c|c|c|c|c|c|c|}
\hline \multirow[b]{2}{*}{ Cause of death } & \multicolumn{2}{|c|}{ All modalities } & \multicolumn{2}{|c|}{ Dialysis } & \multicolumn{2}{|c|}{ Transplant } \\
\hline & $N$ & $\%$ & $N$ & $\%$ & $N$ & $\%$ \\
\hline Cardiac disease & 714 & 22 & 613 & 23 & 101 & 18 \\
\hline Cerebrovascular disease & 138 & 4 & 114 & 4 & 24 & 4 \\
\hline Infection & 688 & 21 & 554 & 21 & 134 & 24 \\
\hline Malignancy & 327 & 10 & 201 & 7 & 126 & 22 \\
\hline Treatment withdrawal & 581 & 18 & 566 & 21 & 15 & 3 \\
\hline Other & 666 & 20 & 534 & 20 & 132 & 24 \\
\hline Uncertain & 144 & 4 & 115 & 4 & 29 & 5 \\
\hline Total & 3,258 & & 2,697 & & 561 & \\
\hline No cause of death data & 1,747 & 35 & 1,439 & 35 & 308 & 35 \\
\hline
\end{tabular}

Table 3.14. Cause of death in prevalent transplant patients on $1 / 1 / 2015$ by age, who died in 2015

\begin{tabular}{|c|c|c|c|c|c|c|}
\hline \multirow[b]{2}{*}{ Cause of death } & \multicolumn{2}{|c|}{ All age groups } & \multicolumn{2}{|c|}{$<65$ years } & \multicolumn{2}{|c|}{$\geqslant 65$ years } \\
\hline & $N$ & $\%$ & $N$ & $\%$ & $N$ & $\%$ \\
\hline Cardiac disease & 101 & 18 & 54 & 21 & 47 & 15 \\
\hline Cerebrovascular disease & 24 & 4 & 13 & 5 & 11 & 4 \\
\hline Infection & 134 & 24 & 58 & 23 & 76 & 25 \\
\hline Malignancy & 126 & 22 & 58 & 23 & 68 & 22 \\
\hline Treatment withdrawal & 15 & 3 & 3 & 1 & 12 & 4 \\
\hline Other & 132 & 24 & 61 & 24 & 71 & 23 \\
\hline Uncertain & 29 & 5 & 10 & 4 & 19 & 6 \\
\hline Total & 561 & & 257 & & 304 & \\
\hline No cause of death data & 308 & 35 & 142 & 36 & 166 & 35 \\
\hline
\end{tabular}

risk group of patients. The leading causes of death amongst transplant patients were malignancy (22\%) and infection (24\%). There has been a reduction over time in the proportion of deaths in transplant patients attributed to cardiovascular or cerebrovascular disease
(43\% in 2003 compared to $22 \%$ in 2015) with an increase in the proportion ascribed to infection or malignancy (30\% in 2003 compared to $46 \%$ in 2015). The increased death rate secondary to malignancy and infection may include the increasing age of transplant recipients and

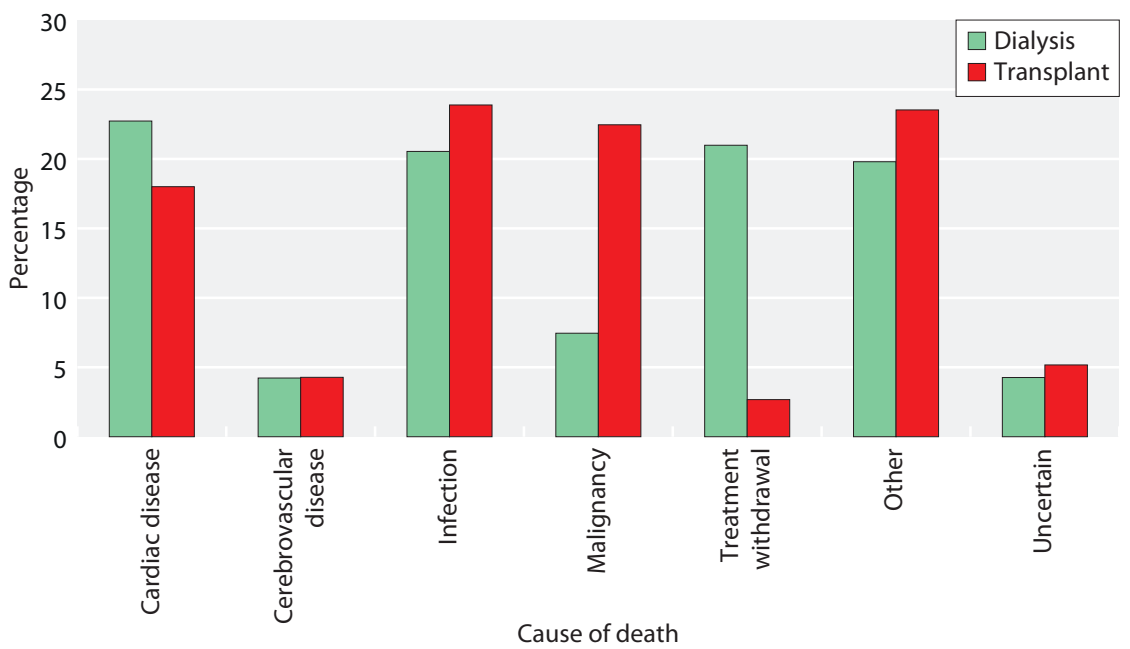

Fig. 3.11. Cause of death by modality for prevalent patients on $1 / 1 / 2015$, who died in 2015 
the increased intensity of immunosuppressive regimens, particularly the use of lymphocyte depleting induction regimes.

\section{References}

1 Ansell D, Tomson CRV: UK Renal Registry 11th Annual Report (December 2008) Chapter 15 The UK Renal Registry, UKRR database, validation and methodology. Nephron Clin Pract 2009;111(suppl 1): c277-c285

2 Pruthi R, Curnow E, Roderick P, Ravanan R. UK Renal Registry 17th Annual Report: Chapter 11 Centre Variation in Access to Renal Transplantation in the UK (2008-2010). Nephron. 2015;129(suppl 1):247-56. doi: $10.1159 / 000370281$.

3 Oniscu GC, Ravanan R, et al. Access to Transplantation and Transplant Outcome Measures (ATTOM); study protocol of a UK wide, in-depth, prospective cohort analysis. BMJ Open 2016; 6: e010377.doi:10.1136/ bmjopen-2015-010377

4 Bosma RJ, Doorenbos CRC, Stegeman CA, Homan van der Heide JJ, Navis G: Predictive Performance of Renal Function Equations in Renal Transplant Recipients: An analysis of Patient Factors in Bias. Am J Transplant 2005;5:2183-2203

5 Froissart M, Rossert J, Jacquot C, Paillard M, Houillier P: Predictive Performance of the Modification of Diet in Renal Disease and Cockcroft-Gault Equations for Estimating Renal Function. J Am Soc Nephrol. 2005; 16:763-773
Conflicts of interest: Dr E Sharples has received travel honoraria from Alexion pharmaceuticals.
6 Hariharan, S, McBride MA, Cherikh WS, Tolleris CB, Bresnahan BA, Johnson CP: Post-transplant renal function in the first year predicts long-term kidney transplant survival. Kidney Int 2002;62:1:311-318

7 UK Renal Association Clinical Practice Guidelines Committee: Anaemia of CKD, 5th Edition. 2010. http://www.renal.org/clinical/Guidelines Section/AnaemiaInCKD.aspx

8 UK Renal Association Clinical Practice Guidelines Committee: Guideline: Post-operative Care of the Kidney Transplant Recipient, 5th Edition. 2011. http://www.renal.org/Clinical/GuidelinesSection/ Post-operative-Care-Kidney-Transplant-Recipient.aspx

9 White CA, Akbari A, Doucette S, Fergusson D, Knoll GA: Estimating Glomerular Filtration Rate in Kidney Transplantation: Is the New Chronic Kidney Disease Epidemiology Collaboration Equation Any Better? Clin Chem 2010;56:3:474-477

10 Ng FL, Holt DW, Chang RWS, MacPhee IAM: Black renal transplant recipients have poorer long-term graft survival than CYP3A5 expressers from other ethnic groups. Nephrol Dial Transplant 2010;25:628-634 


\section{Appendix 1: Reporting status of audit measures}

Table 3.15. The reporting status of the recommended Renal Association Audit Measures for the Post-operative Care of Kidney Transplant Recipients in the 19th Annual Report

\begin{tabular}{ccc}
\hline & Included in \\
RA audit measure & $\begin{array}{c}\text { UKRR annual } \\
\text { report? }\end{array}$ & Reason for non-inclusion \\
\hline
\end{tabular}

1. Proportion of blood results available for review, and reviewed, within 24 hours

2. Proportion of renal centres with a written follow-up schedule available to all staff and patients

3. Percentage of patients accessing their results through Renal Patient View

4. Percentage of total patients assessed in an annual review clinic.

5. Percentage of total patients receiving induction with ILRAs and TDAs

6. Percentage of de novo KTRs receiving tacrolimus

7. Percentage of de novo KTRs receiving MPA based immunosuppression

8. Percentage of de novo KTRs receiving corticosteroid maintenance therapy

9. Use of generic agents

10. Severity of biopsy proven acute rejection (BPAR) recorded by BANFF criteria.

11. Percentage of KTRs with BPAR in first 3 months and first 12 months.

12. Percentage of KTRs requiring TDAs to treat rejection in first year

13. Complication rates after renal transplant biopsy

14. Proportion of patients receiving a target blood pressure of 130/ $80 \mathrm{mmHg}$ or $125 / 75 \mathrm{mmHg}$ in the presence of proteinuria $(\mathrm{PCR}>100$ or ACR $>70)$

15. Proportion of patients receiving an ACE inhibitor or angiotensin receptor blocker

16. Proportion of patients with proteinuria assessed by dipstick and, if present, quantified at each clinic visit.

17. Proportion of renal transplant recipients with an annual fasting lipid profile

18. Proportion of KTR taking statins (including the type of statin) for primary and secondary prevention of premature cardiovascular disease

19. Proportion of patients on other lipid lowering agents

20. Proportion of patients achieving dyslipidaemia targets

21. Incidence of new onset diabetes after transplantation (NODAT) at three months and at annual intervals thereafter

22. Proportion of patients who require insulin, and in whom remedial action is undertaken - minimisation of steroids and switching of CNIs

23. Proportion of patients with ischaemic heart disease

24. Proportion of patients suffering myocardial infarction

25. Proportion of patients undergoing primary revascularisation
No

No

No

No

No

No

No

No

No

No

No

No

No

No

No

No

No

No

No

Partly

No

No

No

No

No
UKRR does not currently collect these data UKRR does not currently collect these data Requires linkage with RPV

UKRR does not currently collect these data Poor data completeness

Poor data completeness

Poor data completeness

Poor data completeness

UKRR does not currently collect these data UKRR does not currently collect these data

UKRR does not currently collect these data

UKRR does not currently collect these data UKRR does not currently collect these data Poor data completeness on proteinuria

Poor data completeness

UKRR does not currently collect these data

UKRR does not currently collect these data

UKRR does not currently collect these data

Poor data completeness

Reported but not a centre level, but by transplant status

UKRR does not currently collect these data

UKRR does not currently collect these data

Poor data completeness

Poor data completeness

Poor data completeness 
Table 3.15. Continued

Included in

UKRR annual

RA audit measure

26. Proportion of patients receiving secondary prevention with a statin, anti-platelet agents and RAS blockers

27. Proportion of patients who are obese

28. Proportion of patients having screening procedures for neoplasia at the annual review clinic

29. Incidence of CMV disease

30. Rate of EBV infection and PTLD

31. Completeness of records for EBV donor and recipient serology

32. Rates of primary VZV and shingles infection

33. Completeness of records for VZV recipient serology

34. Rates and outcomes of HSV infection.

35. Rates of BK viral infection in screening tests.

36. Rates and outcomes of BK nephropathy

37. Frequency of bisphosponate use

38. Incidence of fractures

39. Incidence of hyperparathyroidism

40. Incidence of parathyroidectomy

41. Use of cinacalcet

42. Frequency of hyperuricaemia and gout

43. Prevalence of anaemia

44. Prevalence of polycythaemia

45. Pregnancy rates and outcomes

46. Prevalence of sexual dysfunction report?

Reason for non-inclusion

No UKRR does not currently collect these data

No Poor data completeness

No UKRR does not currently collect these data

No Poor data completeness

No UKRR does not currently collect these data

No UKRR does not currently collect these data

No UKRR does not currently collect these data

No UKRR does not currently collect these data

No UKRR does not currently collect these data

No UKRR does not currently collect these data

No UKRR does not currently collect these data

No UKRR does not currently collect these data

No UKRR does not currently collect these data

Partly Reported but not a centre level, due to poor data completeness

No UKRR does not currently collect these data

No Poor data completeness

No UKRR does not currently collect these data

Yes

No Poor data completeness

No UKRR does not currently collect these data

No UKRR does not currently collect these data 